\title{
3D Sensors for Sewer Inspection: A Quantitative Review and Analysis
}

\author{
Chris H. Bahnsen *(D), Anders S. Johansen (D), Mark P. Philipsen ${ }^{(D}$, Jesper W. Henriksen $(\mathbb{D}$, Kamal Nasrollahi $(\mathbb{D})$ and \\ Thomas B. Moeslund (D)
}

check for updates

Citation: Bahnsen, C.H.; Johansen, A.S.; Philipsen, M.P.; Henriksen, J.W.; Nasrollahi, K.; Moeslund, T.B. 3D Sensors for Sewer Inspection: A Quantitative Review and Analysis. Sensors 2021, 21, 2553. https:// doi.org/10.3390/s21072553

Academic Editor: Jochen Lang

Received: 17 February 2021

Accepted: 2 April 2021

Published: 6 April 2021

Publisher's Note: MDPI stays neutral with regard to jurisdictional claims in published maps and institutional affiliations.

Copyright: (c) 2021 by the authors. Licensee MDPI, Basel, Switzerland. This article is an open access article distributed under the terms and conditions of the Creative Commons Attribution (CC BY) license (https:// creativecommons.org/licenses/by/ $4.0 /)$.
Visual Analysis and Perception (VAP) Lab, Aalborg University, Rendsburggade 14, 9000 Aalborg, Denmark; asjo@create.aau.dk (A.S.J.); mpph@create.aau.dk (M.P.P.); jwhe@create.aau.dk (J.W.H.); kn@create.aau.dk (K.N.); tbm@create.aau.dk (T.B.M.)

* Correspondence: cb@create.aau.dk

\begin{abstract}
Automating inspection of critical infrastructure such as sewer systems will help utilities optimize maintenance and replacement schedules. The current inspection process consists of manual reviews of video as an operator controls a sewer inspection vehicle remotely. The process is slow, labor-intensive, and expensive and presents a huge potential for automation. With this work, we address a central component of the next generation of robotic inspection of sewers, namely the choice of 3D sensing technology. We investigate three prominent techniques for 3D vision: passive stereo, active stereo, and time-of-flight (ToF). The Realsense D435 camera is chosen as the representative of the first two techniques wheres the PMD CamBoard pico flexx represents ToF. The 3D reconstruction performance of the sensors is assessed in both a laboratory setup and in an outdoor above-ground setup. The acquired point clouds from the sensors are compared with reference 3D models using the cloud-to-mesh metric. The reconstruction performance of the sensors is tested with respect to different illuminance levels and different levels of water in the pipes. The results of the tests show that the ToF-based point cloud from the pico flexx is superior to the output of the active and passive stereo cameras.
\end{abstract}

Keywords: sewer inspection; sewer pipes; 3D vision; 3D reconstruction; computer vision; automated inspection

\section{Introduction}

Sewer networks are a critical piece of infrastructure that enable the proper disposal of wastewater and rain water in modern societies. Even though the uphold of sewers is instrumental to our modern way of living, their location in the underground means that the assessment and maintenance of the sewer infrastructure is often overlooked or postponed. The ubiquitous nature of sewers is confirmed by the raw numbers; in the US, the extent of the public sewer network are more than 1.2 million $\mathrm{km} \mathrm{[1]} \mathrm{and} \mathrm{in} \mathrm{Germany,} \mathrm{there} \mathrm{are} \mathrm{more}$ than $594,000 \mathrm{~km}$ of sewage and rain water pipes [2]. As the sewer pipes age, so does the likelihood of damage or leakage of the pipes. In order to prevent an untimely breakdown and replacement of a pipe, rehabilitation works may be carried out on older networks. As more than 70,000 km of the German sewage pipes were built before 1960 [2], there is a need for timely inspection of the sewer network in order to assess the condition of the pipes. However, approximately $14 \%$ of the pipes are not accessible with current inspection techniques [3] and only 13\% of the responding authorities in a German survey can approve the current state of their sewer systems [3]. There is thus a need for further rehabilitation and renovation works in the sewer systems. The allocation of resources for such works is guided by inspections of the current state of the sewer systems.

Currently, sewer inspections are carried out by trained professionals who manually operate a custom-built tethered inspection vehicle that is inserted from a well into the sewer pipe [4]. The human operator controls the vehicle remotely from above ground and 
manually annotates the conditions of the pipe by inspecting the Closed-Circuit Television (CCTV)-footage that is streamed from the vehicle. In order to decrease the risk of the tether getting stuck in the pipe, the inspection vehicle only drives from one well to another before being extracted and inserted into the next well. Effort has been put into automating parts of the process such as the automatic locomotion of the robot [5] or the automatic annotation of the image data provided by the CCTV-footage of the robot [6].

The vision of a fully autonomous robot for sewer inspection has spurred many interesting research projects over the last 25 years which have produced several robotic prototypes for demonstration purposes such as KURT [7], MAKRO [8], KANTARO [9], ARSI [10], and SIAR [11]. For a full overview, we refer to the excellent survey by Mirats-Tur and Garthwaite [12]. The focus of the research projects listed above is mainly on the challenging task of building a robotic prototype that can navigate within the confined space of a sewer network whereas less attention has been devoted into the design and test of the sensing capabilities of said robots. There is a need, however, to study the proper selection of sensors that should guide the autonomous sewer inspection robots of the future. As evidenced by Haurum and Moeslund [6], there is increasing interest in the research community for automating the annotation process of the images extracted from the CCTV-footage of the inspection vehicles.

However, a fully autonomous, untethered robot faces a tough navigation challenge when it must traverse the entire sewer system of a neighbourhood or an entire city. This includes keeping track of the position of the robot in an Global Navigation Satellite System (GNSS)-denied environment and safely maneuvering the robot in a hostile environment. It is therefore necessary to move from two-dimensional image-based inspection towards three-dimensional (3D) depth-based inspection guided by guided by recent advances in robust, low-powered depth sensors. Although such sensors have been applied in recent projects on autonomous sewer inspection robots $[10,11]$, their depth sensing qualities have not been thoroughly assessed.

The focus of this work is to study the characteristics of 3D sensors that can be attached on a future autonomous robotic platform for sewer inspection. In particular, we study how the choice of $3 \mathrm{D}$ sensors affect the performance of $3 \mathrm{D}$ reconstruction of the sewer pipe which is important for the condition assessment of the sewer pipe and navigation and localization capabilities of the autonomous robot. An autonomous robot should not be hindered by any tethering to the surroundings. However, untethered robots are constrained with respect to battery power and capacity. Maintaining a sufficient lit environment in order to sense the surroundings may draw a lot of power, and it might thus be interesting to study how the presence or absence of light will impact the accuracy of the 3D sensors.

In this work, we will therefore study the influence of light on the accuracy of 3D sensors within the context of sewer inspection. Furthermore, we will study how the presence of water in the sewer pipes affects the capabilities of depth sensors. Our contributions are as follows:

- We simulate the sewer environment using two different setups: a clean laboratory environment with reflective plastic pipes and an outdoor above-ground setup with four wells connected by pipes with different diameters and topology.

- We utilize the laboratory and above-ground setup to systematically test passive stereo, active stereo, and time-of-flight 3D sensing technologies under a range of different illuminance levels.

- The laboratory setup is utilized for assessing how active stereo and time-of-flight sensors are affected by the presence of various levels of water in the sewer pipe.

- We systematically evaluate the reconstruction performance of the 3D sensors from the experiments and compare it with reference models of the sewer pipes.

The remainder of the paper is organised as follows: Section 2 presents an overview of related work within 3D sensing of sewers and pipe-like environments. Section 3 gives an overview of suitable depth sensing technologies for inspection of sewers. Section 4 describes the experimental setup and methods used for the illumination tests and water 
level tests. Section 5 analyses the experimental results, and Section 6 concludes on the results and gives directions for further investigation.

\section{Related Work}

The need to autonomously navigate, map and inspect hazardous environments has in recent years led to increasing interest for implementing 3D vision techniques to create a dense representation of the surroundings. On the MAKRO robot prototype in the late 90s, a laser projector is combined with a rotating mirror to map the distances to the upper sections of the sewer pipe [13]. A similar method is used on the KANTARO robot where the infrared range scanner is located on a protruding stick at the back of the robot [9].

In order to map the scope of 3D vision techniques for compact environments, a systematic literature study has been conducted. Seven scholarly databases have been searched for related works in the field of sewer and pipe inspection. The search was constrained to articles in which title, abstract, or keywords contained one entry from each of the terms in the list below:

- Sewer OR pipe;

- 3D;

- Inspection OR reconstruction OR assessment.

The number of articles from the search of the scholarly databases is visible from the first column of Figure 1. The databases of Google Scholar and ASCE Library are excluded based on the inability to constrain the search to title, abstract, and keywords. Every record from the databases is manually screened based on the title and abstract of the record. From this stage, records that do not consider in-pipe analysis are filtered out alongside records that do not contain any sensing component. After the screening stage, the records from all the databases are combined and duplicates are removed. The full-text content of the remaining 59 articles is assessed of which 13 articles are removed based on the above criteria.

The remaining 46 articles have been assessed and categorized based on the sensors that they use to perceive the environment. The findings are summarized in Table 1 where they are located amongst other references found outside the systematic literature study. The related works are categorized into five groups in Table 1 based on the utilized depth sensing hardware.

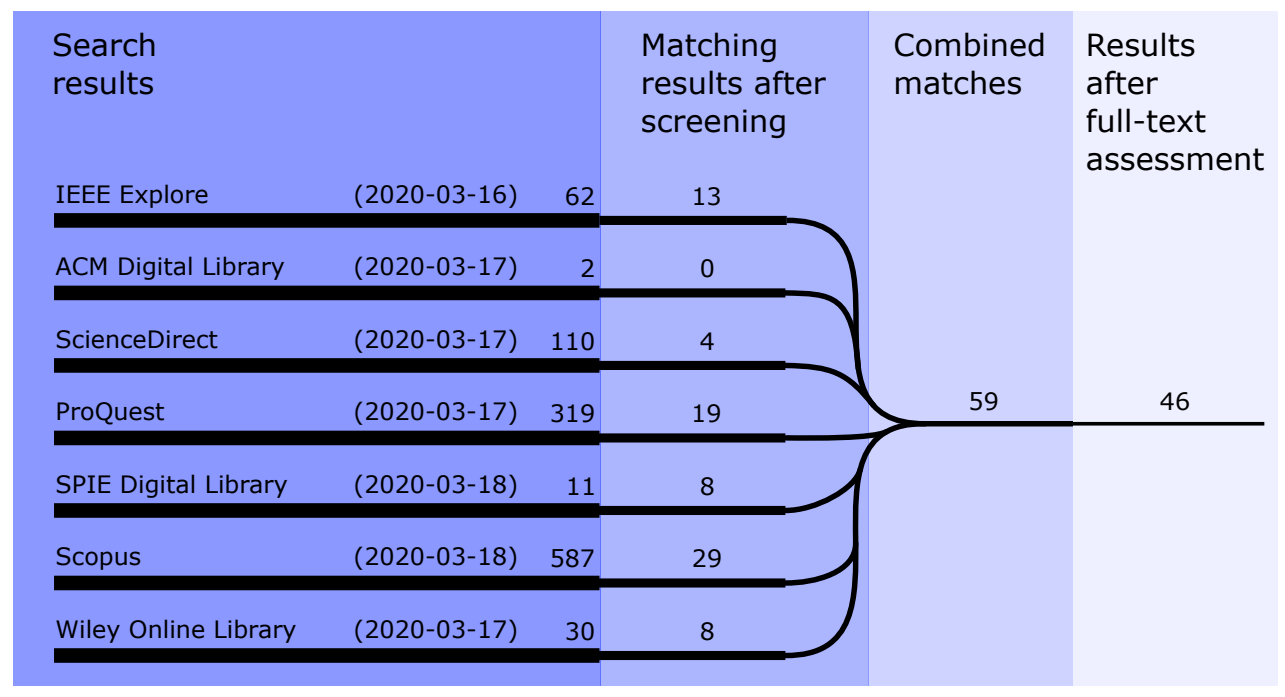

Figure 1. Flow of the literature search. The records of the seven scholarly databases are manually screened based on the title, abstract, and keywords. In a subsequent stage, the records are combined and checked for duplicates. In the final process (light blue), the full-text of every record is manually assessed. 
Table 1. Overview of related work on depth sensing technology for internal inspection of sewers or pipe networks. The depth sensing hardware of each paper is categorized into four categories; Camera if the setup contains any camera, Stereo if the setup contains a passive or active stereo setup, Laser if the setup contains a one-dimensional (rotating) laser, and omnidirectional if the setup either contains a catadioptric sensor or a camera with a fish-eye lens.

\begin{tabular}{|c|c|c|c|c|c|c|c|}
\hline Paper & Year & Camera & Stereo & Laser & $\begin{array}{l}\text { Omni- } \\
\text { Directional }\end{array}$ & $\begin{array}{l}\text { Other } \\
\text { Sensors }\end{array}$ & Reconstruction Technique \\
\hline [14] & 1995 & & & $x$ & & & Laser profilometry \\
\hline$[13,15]$ & 1998 & $x$ & & $x$ & & Ultras. & Laser profilometry \\
\hline [16] & 1998 & $x$ & & $x$ & & & Laser profilometry \\
\hline [17] & 1999 & $X$ & $X$ & & & & None \\
\hline [18] & 2000 & $x$ & & & & & Image proc. + Hough transform \\
\hline [19] & 2000 & $x$ & & $x$ & & & Image proc. + Hough transform \\
\hline [20] & 2002 & $x$ & $x$ & & & & None \\
\hline [21] & 2003 & & & & & LiDAR & ICP \\
\hline [22] & 2003 & $x$ & & & & & Laser profilometry \\
\hline [23] & 2005 & $x$ & & $X$ & $x$ & & Laser profilometry \\
\hline [24] & 2007 & & & $x$ & & & Laser profilometry \\
\hline [9] & 2007 & $x$ & & $\mathrm{X}$ & $x$ & & Laser profilometry \\
\hline [25] & 2007 & $x$ & & & $x$ & & $\mathrm{SfM}+$ cylinder fitting \\
\hline [26] & 2008 & $x$ & & $x$ & & & Laser profilometry \\
\hline [27] & 2009 & $x$ & & & & & Sparse 3D + mosaicing \\
\hline$[28,29]$ & 2009 & $x$ & & & $x$ & & SfM \\
\hline [30] & 2010 & $x$ & $x$ & $x$ & & Ultras. & Image proc. + feat. match. \\
\hline [31] & 2011 & $x$ & $x$ & & & & Dense stereo matching \\
\hline [32] & 2011 & $x$ & & & $x$ & & Dense stereo matching \\
\hline [33] & 2013 & & & $x$ & & & Laser profilometry \\
\hline [34] & 2013 & $x$ & & $x$ & & & Laser profilometry \\
\hline [35] & 2013 & $x$ & & $\mathrm{X}$ & & & Laser profilometry \\
\hline [36] & 2014 & $x$ & & & & LiDAR & $\mathrm{ICP}$ \\
\hline [37] & 2014 & $x$ & & $x$ & & & Structured light \\
\hline [38] & 2014 & $x$ & & $X$ & & & Dense stereo matching \\
\hline [39] & 2014 & $x$ & & & & & Fourier image correspond. \\
\hline [40] & 2014 & & & $x$ & & & Commercial laser scanner \\
\hline [41] & 2014 & $x$ & & & & ToF & Hough transf. (cylinder) \\
\hline [42] & 2015 & $x$ & $x$ & & & Kinect & RGB-D SLAM \\
\hline [43] & 2015 & $x$ & & $X$ & & & Structured light + sparse 3D \\
\hline [44] & 2015 & $x$ & $x$ & $x$ & $x$ & & Laser profilometry \\
\hline [45] & 2015 & $x$ & $x$ & & & & Dense stereo matching \\
\hline [46] & 2016 & $x$ & $x$ & & & & Dense stereo matching \\
\hline [47] & 2016 & $x$ & & $x$ & & & Laser profilometry \\
\hline [48] & 2017 & $x$ & & $x$ & & & Laser profilometry \\
\hline [49] & 2017 & $x$ & $x$ & & & LiDAR & Dense stereo matching \\
\hline [50] & 2018 & $x$ & $x$ & $X$ & $x$ & & Laser profilometry \\
\hline [51] & 2018 & $x$ & $x$ & & & ToF & Visual inertial odometry \\
\hline [52] & 2019 & $x$ & & & & & Dense stereo matching \\
\hline [53] & 2019 & $x$ & & & $x$ & & Dense stereo matching $+\mathrm{SfM}$ \\
\hline [54] & 2019 & $x$ & & $x$ & & & Laser profilometry \\
\hline [55] & 2019 & $x$ & & $x$ & $x$ & & Laser profilometry \\
\hline [56] & 2019 & $x$ & & & & & Dense stereo matching \\
\hline [57] & 2019 & $x$ & & & & LiDAR & Radius estimation from curvature \\
\hline [58] & 2019 & $x$ & & & $x$ & & Dense stereo matching \\
\hline [59] & 2020 & $x$ & $x$ & $x$ & & LiDAR & RGB-D SLAM \\
\hline [60] & 2020 & $x$ & $x$ & & & Kinect & ICP + visual SLAM \\
\hline [61] & 2020 & $x$ & $x$ & & & LiDAR & ICP \\
\hline [62] & 2020 & $x$ & $x$ & & & & Dense stereo matching \\
\hline
\end{tabular}

\subsection{Laser-Based Sensing}

One of the most commonly used techniques for sensing the geometry of the pipe is by the use of laser profilometry. A rotating laser beam projects a ring inside the pipe and a camera is positioned such that it captures the full extent of the ring. By extracting the contour of the laser ring from the image, the cross section of the pipe can be measured, and any imperfections from the elliptical ring may be classified as defects or corrosion. A variant of this setup is the use of multiple fixed, non-rotating laser diodes such as in the work of Hansen et al. [43]. The positions of the laser diodes are tracked through consecutive 
frames, and the tracking enables estimation of the metric radius of the pipe. In [19], a crosshair laser beam is projected ahead of the inspection vehicle in order to guide the navigation and motion planning.

The conceptual simplicity of the laser-based methods is one of its virtues as a tool for an autonomous inspection robot. The cross section of the pipe is easy to interpret, and the detection of structural defects is straightforward. On the other hand, laser profilometry only scans the surface in one plane that moves along the pipe as the robot traverses. This implies that a full 3D reconstruction of the pipe is available only after the robot has moved through the entire pipe. However, an online 2D view of the pipe is not usable in order to navigate a fully autonomous inspection robot in a possibly unknown environment.

\subsection{Omnidirectional Vision}

Omnidirectional vision sensors allow for a full view of the internal surface of the pipe. The full view is either produced by a catadioptric camera system $[23,44,50,55,58]$ or by the use of fish-eye lenses $[9,25,28,29,32,53]$. Omnidirectional vision sensors provide a view of the entirety of the pipe with just a single sensor and are used in combination with laser profilometry $[9,23,44,50,55]$ or stereo matching $[25,29,32,53,58]$ approaches.

Compared to traditional cameras, the full view comes at the cost of either complicated hardware in the case of catadioptric cameras and additional post-processing of the acquired imagery for both solutions. With careful calibration of the camera systems, however, a very detailed, wide-angle view of the pipe may be acquired.

\subsection{Stereo Matching}

A dense 3D reconstruction of the pipe may be provided by the use of stereo matching techniques with either monocular or stereo cameras. With monocular cameras, cylindrical or elliptical [25] constraints are often imposed in order to guide the reconstruction. The addition of an additional camera enables the computation of structure independent of the motion of the robot and allows for the relaxation of the cylindrical constraints. Two-view stereo matching is applied in a sewer context by Huynh et al. $[45,46]$ to detect cracks and defects in sewers. While their experiments show that it is a viable strategy, they also note that their method has issues detecting small defects or thin cracks that are hard to locate in both camera views. A common problem with traditional passive stereo, they had problems with matching features between the two views in case of very uniform environments. Furthermore, stereo matching is a computationally heavy operation that imposes high processing requirements in order to retrieve a high-quality point cloud.

An approach to combating the feature matching problem is the use of active stereo techniques. Active stereo techniques project a set of distinct features onto the surface. The active stereo camera is combined with an Inertial Measurement Unit (IMU) for effective localization in the context of drone-based 3D cave mapping [63]. A similar approach is found in [51] for mapping of underground tunnels. The authors have replaced the active stereo depth sensor with a time-of-flight $(\mathrm{ToF})$ sensor while also using monochrome Charged Coupled Device (CCD) cameras in a stereo setup. Utilizing CCD instead of Complementary metal-oxide-semiconductor (CMOS) based cameras allows them to capture images with a global shutter so light only has to be emitted from the robot the moment it takes the picture, reducing potential interference the reflected light could have on the ToF sensor.

\subsection{Other Sensing Approaches}

The recent work of Kolvenbach et al. [61] within inspection of sewers utilizes active stereo for a dense 3D map of the local environment while relying on a Velodyne VLP16 LIDAR to do a long range, rough mapping of the environment for localization and navigation. The Kinect $\mathrm{v} 1$ and Kinect $\mathrm{v} 2$ cameras are used in [57] to estimate the radius of pipes. The high-end FARO LS $840 \mathrm{HE}$ laser scanner is used to build a reference model with which the much more affordable Kinect devices are compared. 


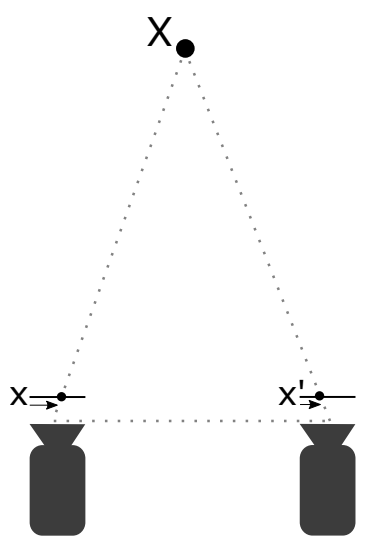

(a) Passive stereo vision.

\section{Depth Sensors}

Depth sensing technology is improving at a rapid pace. The number of sensors that are available has exploded while prices have reached a customer friendly level. Overall, depth sensing technologies fall into three categories: stereo vision, ToF, and depth from monocular cues.

Stereo-based methods rely on the parallax or displacement that occurs when an object $X$ is viewed from different points of view. This is illustrated by pixel $x$ and $x^{\prime}$ in the image planes shown in Figure 2a,b, respectively. Objects close to the cameras have a greater disparity in position between the different views than objects that are further away. The depth of an object seen from both views may be computed from the disparity by the use of similar triangles and the prior knowledge of the baseline and focal length of the stereo setup. The hard problem is identifying the same locations in both views in order to calculate the disparity. Passive stereo cameras consist of two cameras that monitor the same scene from different but well known points of view. For matching algorithms to successfully identify features and calculate disparities, the scene must contain distinct features. Active stereo, as illustrated in Figure 2b, adds a projected light pattern to the scene in order to perform better in low texture environments. Otherwise, the method works in the same way as a passive stereo camera. Depth from structured light, as illustrated in Figure 2c, also projects light patterns into the scene. But instead of using two cameras, knowledge of the transmitted pattern is used to perform triangulation using a single camera and the projector. Because this method relies on recognizing a well known projected pattern, it is sensitive to external interference. All are reliant on the known relationship between the two viewpoints, making the structural integrity of the setup crucial. The three variations of stereo vision are illustrated in Figure 2.

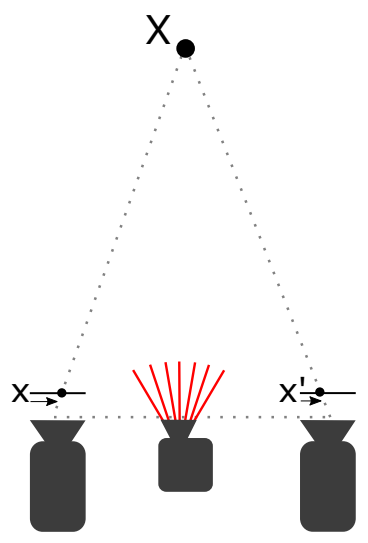

(b) Active stereo vision.

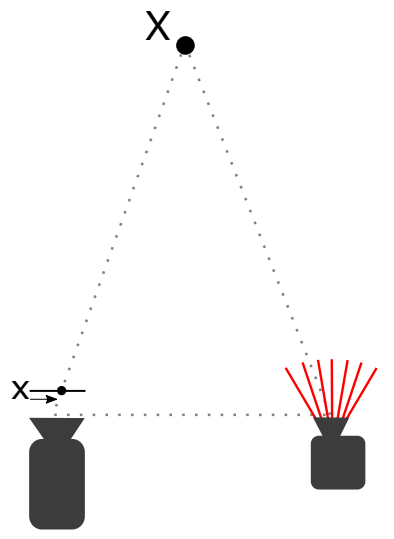

(c) Structured light stereo vision.

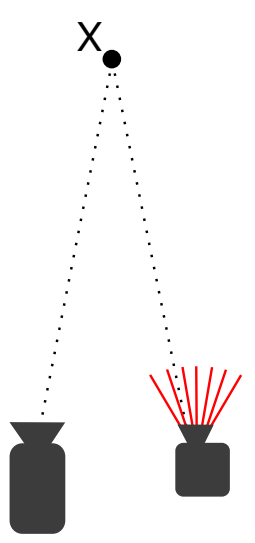

(d) Time-of-Flight.
Figure 2. Different variations of depth sensing techniques.

ToF sensors measure distance based on the time it takes emitted light to be reflected by an object in the scene and return to an image sensor. This is illustrated in Figure $2 \mathrm{~d}$. Achieving a usable depth resolution with this method requires very high clock frequencies from the hardware. Because depth measurement does not rely on triangulation, however, ToF cameras can be made as small as the sizes of the laser and sensor allow.

Recently, advances within machine learning algorithms have shown impressive results for estimating depth from monocular vision. This is possible because visual cues such as focus, sizes, shadows, perspective, motion, etc. are correlated with depth. Since ground-truth depth data that can be used to train such algorithms is prohibitively difficult to acquire, self-supervised approaches to learning depth estimation are very enticing. Clément et al. [64] make use of the known relationship between stereo image pairs when training a CNN to estimate disparity. The right image of a stereo pair can be approximated 
from the left image of the same stereo pair based on the calculated disparity. Thus, a loss function can be designed to minimize the differences between the image pair given the estimated disparity by a CNN.

Monocular depth estimation can be learned using stereo pairs as described above or from monocular video. Using monocular video requires ego-motion estimates between temporal image pairs. Clément et al. [65] propose a number of tricks that improve monocular depth estimation and show how self-supervision from both video and stereo pairs can be combined. These methods may fail to correctly estimate depth for images that are distorted, contain reflections or are highly detailed.

We select the sensors for comparison in this work based on their popularity and representability of the underlying technologies. Due to the low popularity of structured light and the poor performance that is expected for this technology under less controlled conditions, structured light is eliminated up-front. It is safe to say that the Microsoft's Kinect depth sensors have been the most widely used in 3D vision research. For this reason, Azure Kinect (Microsoft Corporation, Redmond, WA, United States), the successor to the popular Kinect 2, is an obvious candidate for our case. It is capable of acquiring accurate high resolution point clouds using ToF technology and can be optimized for a wide or narrow field of view depending on the application. The Kinect family has already been thoroughly covered by Tölgyessy et al. [66] where performance is measured across different surface types. Their research shows that surfaces at the extremes of the reflective-absorbent spectrum are most challenging. Out of the box, Azure Kinect is too fragile with an operational temperature range of $10-25^{\circ} \mathrm{C}$. Mitigating this problem will add significantly to the complexity and size of the payload. Considering the limited payload of a sewer inspection robot, PMD CamBoard pico flexx (PMD Technologies AG, Siegen, Germany), or pico flexx for short, with its small size and low weight of $8 \mathrm{~g}$, is a good choice as representative of ToF sensors. It has previously been used for indoor localization and mapping in both light and dark environments [67] and for exploration and mapping of tunnels [51]. The selection of stereo cameras is large, but when limited to smaller cameras, the RealSense D400 family of stereo cameras is probably the most popular. With a short to medium size baseline and a global shutter, the RealSense D435 (Intel Corporation, Santa Clara, CA, United States)is the best choice for sewer inspection. It is used in the ARSI autonomous micro air vehicle for inspection of sewers [10] and for aerial robotic navigation in low-light GNSS-denied conditions [68]. Because it contains a NIR laser projector it can function as either a passive or active stereo camera and lets us cover both passive and active stereo vision using a single camera. Table 2 lists some of the central specifications of the two cameras.

Table 2. Specifications of the chosen pico flexx [69] and RealSense D435 cameras [70].

\begin{tabular}{|c|c|c|c|c|c|c|}
\hline Camera & Resolution (Pixels) & Frame Rate (fps) & Range (m) & Power (w) & Size $(\mathrm{mm})$ & Weight (g) \\
\hline PMD CamBoard pico flexx & $224 \times 171$ & 45 & $0.1-4$ & $\approx 0.3$ & $68 \times 17 \times 7.25$ & 8 \\
\hline RealSense D435 & $1920 \times 1080$ & 30 & $0.3-3$ & $<2$ & $90 \times 25 \times 25$ & 9 \\
\hline
\end{tabular}

\section{Materials and Methods}

The Realsense and pico flexx depth sensors that have been selected as representatives of the different relevant depth sensing technologies and as potential sensors for a sewer inspection robot platform are tested in order to assess and compare their suitability for inspection of sewer pipes.

The tests serve two purposes, namely, to investigate the sensitivity of the sensors with respect to

- Different illuminance levels in the pipe;

- Different water levels in the pipe.

The illumination tests are conducted both in a laboratory and in an above-ground outdoor test setup whereas the water level test is conducted solely in the laboratory. 


\subsection{Robotic Setup}

The depth sensors are integrated on a purpose-built LEGO platform and connected to a Raspberry Pi 4 with 4 GB of RAM (Raspberry Pi Foundation, Cambridge, United Kingdom). Sensor data are recorded directly on the Raspberry Pi and subsequently transferred to a PC for analysis.

The illumination of the sewer pipe is provided by two different light sources; a custombuilt ring light for the laboratory experiments and an industrial-grade brick light [71] for the outdoor experiments. The robotic setups for both cases are shown in Figure 3.

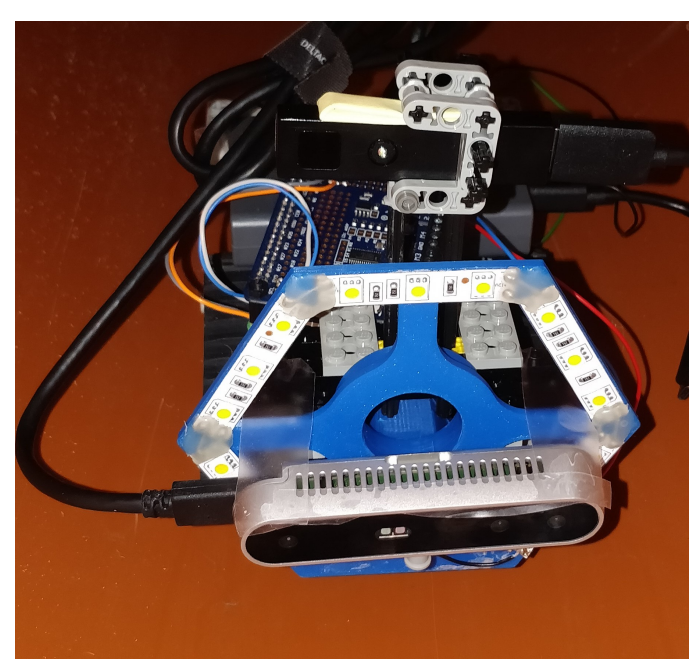

(a) Robot for laboratory experiments.

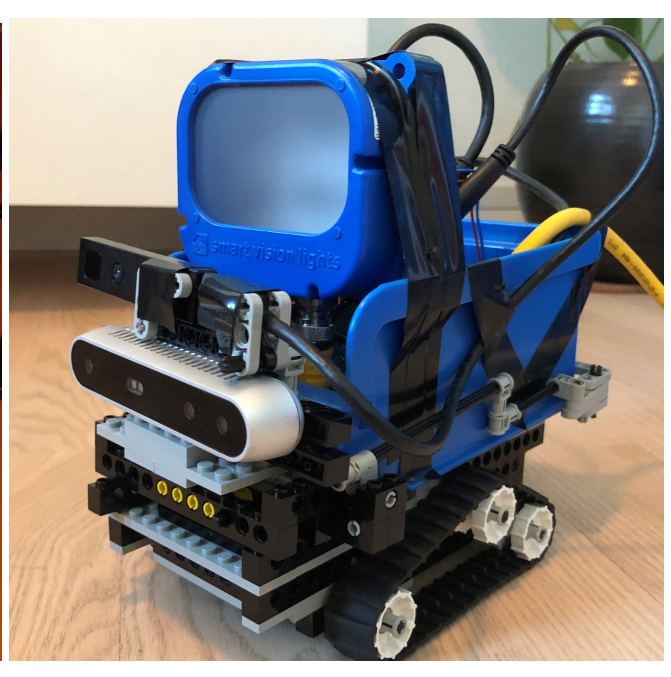

(b) Robot for outdoor experiments.

Figure 3. Robotic setup for the laboratory and outdoor experiments. The setup is upgraded for the outdoor experiments with a new light source and a blue box that protects the Raspberry Pi from dirt.

As noted in Section 3, the Realsense camera contains an infrared emitter that can be turned on and off. When turned off, the Realsense works as a traditional passive depth sensor, relying only on the ambient light for estimating the depth of objects. When the infrared emitter is turned on, the camera may operate without any external light source. These two modes of operation are tested for the illumination tests whereas only the default mode with the emitter turned on is tested for the water level experiments. Any other setting on the Realsense camera is set as default. The pico flexx camera comes with a variety of work modes. For these experiments, the camera is configured with "Mode 9" that enables a frame rate of 15 frames/second, which results in a reported depth range of $0.5-1.5 \mathrm{~m}$ [72]. The configuration is a trade off between range, accuracy, and frame rate. It is assumed that the sewer robot will move slowly in the sewer pipes, which means that a frame rate of 15 frames/second is sufficient. The chosen configuration is the one that matches an acceptable accuracy with a minimum depth range that allows the robot to see objects within a reasonable driving range.

\subsection{Assessing the Point Clouds}

The raw output of the illumination tests and water level tests is a sequence of point clouds from each sensor. Methods for assessing the quality of the point clouds may be grouped into two broad categories: quantitative analysis by comparing the point clouds with a reference model and human perception for judging the visual traits of the point clouds. The following provides an overview of techniques for performing quantitative analysis of point clouds:

Point-to-Point The distance to the nearest neighbor is found by measuring distances between a given point and every point in a reference point cloud. This is illustrated in Figure 4a where the closest point in the reference point cloud is found, and distance $d$ corresponds to the matching error for the point pair marked with green. 
Point-to-Plane The method estimates the surface of the reference point cloud. The surface of a point in the reference cloud is computed using the neighborhood points around the matching point in the reference cloud. In Figure $4 b$, the neighborhood is illustrated by the gray oval, and the estimated surface is represented by the dotted red line. The vector between the point-to-point match connected by $d$ is projected onto the surface of the reference cloud. Together, the point-to-point vector $d$ and the vector $d_{p}$ projected onto the reference surface are used to compute an error vector $d_{e}$ that is normal to the estimated surface in the reference cloud [73].

Point-to-Mesh Distances between a point cloud and a reference mesh are defined as either the orthogonal distance from a point in the measured cloud to the triangular plane in the reference mesh or as the distance is to the nearest edge, in case the orthogonal projection of the point falls outside the triangle [74]. The two possibilities are illustrated in Figure 4c.

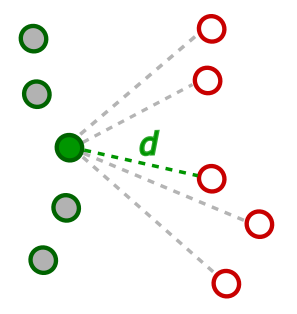

(a) Point-to-Point.

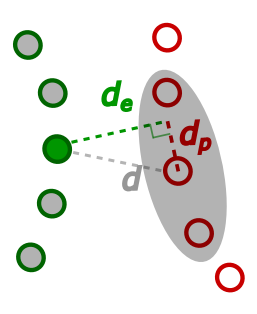

(b) Point-to-Plane.

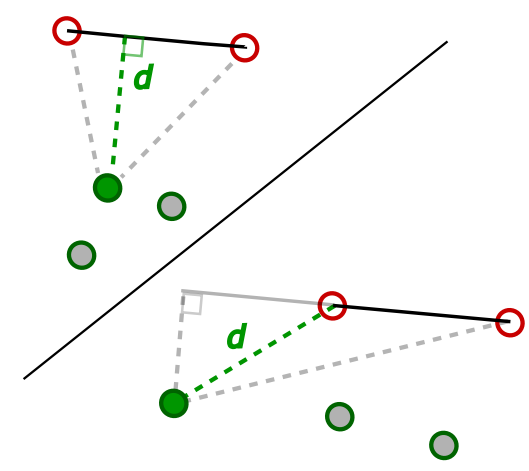

(c) Point-to-Mesh.

Figure 4. Three different types of point cloud comparison. Red markings signify the reference, while green and gray signify the measured object.

Alternatively, human perception can be used to compare and judge point clouds [75]. This requires time and calibration and is likely to produce highly subjective results. The approach is well suited for cases where the point cloud is intended for human consumption or when an algorithmic approach is not feasible. Besides its uses in evaluation of data acquisition systems, comparison and quality assessment of point clouds is used in the evaluation of denoising [73] and compression [76] methods and used in methods for measuring of terrestrial surface changes due to erosion, sedimentation, growth, and construction [77].

As stated in the Introduction, the point clouds generated from the depth sensors should assist an autonomous inspection robot in reconstructing the sewer pipe in order to detect defects, map the topology of the pipe, and determine drivable sections of the pipe. In order to complete these tasks, it is important that the acquired point cloud be an accurate representation of the real pipe. As the experiments are formed in mostly flush pipes, it is a fair approximation to model the pipe as a geometric cylinder with a diameter that resembles the interior diameter of the pipe. The geometric cylinder as a reference model also provides a mesh that can be used for computing distances from the cylindrical surface to the point cloud. This enables the use of the point-to-mesh method mentioned above. The comparison of the acquired point cloud to the reference mesh of a cylinder is performed as follows:

1. A point cloud is constructed from each depth image by back-projecting the depth points by the use of the intrinsic parameters from each camera.

2. A 3D model of the sewer pipe is loaded into the CloudCompare software toolbox [78]. The 3D model is made such that it matches the interior diameter of the sewer pipe.

3. The point cloud of each sensor is loaded into CloudCompare and cropped if necessary. 
4. The loaded point cloud from the sensors are likely displaced and rotated with respect to the 3D model which means that they cannot be directly compared. There are two methods for aligning the point cloud to the 3D model:

(a) Directly measuring the rotation and displacement of the depth sensor with respect to the pipe.

(b) Estimating the transformation by proxy methods that works directly on the point cloud.

5. For these experiments, method (b) is chosen as it was not possible to directly measure the position of the sensor with the required accuracy. A RANSAC-based method [79] available as a plug-in to CloudCompare is used to estimate cylinders from the point cloud of each sensor. If the reconstructed point cloud is noisy, the RANSAC estimation might produce several cylinders. The cylinder that resembles the physical pipe is selected manually. The affine transformation that relates the cylinder to the origin of the coordinate frame is obtained.

6. The inverse of the transformation obtained from step 5 is used to align the point cloud to the 3D model, assuming that the 3D model is centered at $(0,0,0)$, that the circular cross-section of the cylindrical model is parallel to the XY-plane, and that the height of the cylinder is expanding along the $z$-axis.

7. The distance between the point cloud from the sensor and the 3D model is computed by using the "Cloud to mesh distance" tool from CloudCompare. The distance is stored as a scalar field in a new point cloud. Sample distance measurements from the Realsense and pico flexx depth sensors are shown in Figure 5.

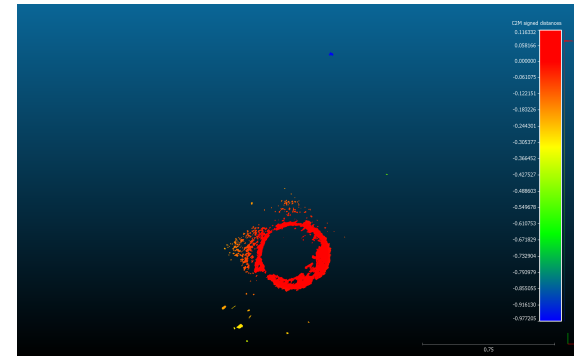

(a) Realsense emitter off

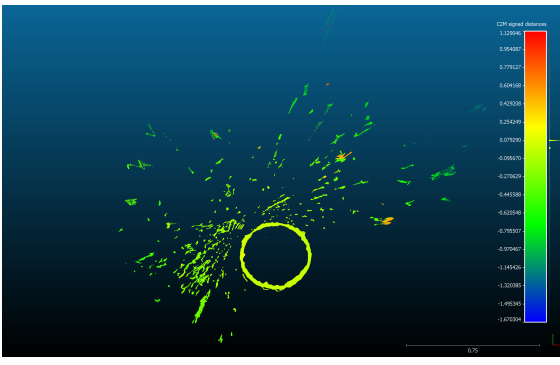

(b) Realsense emitter on

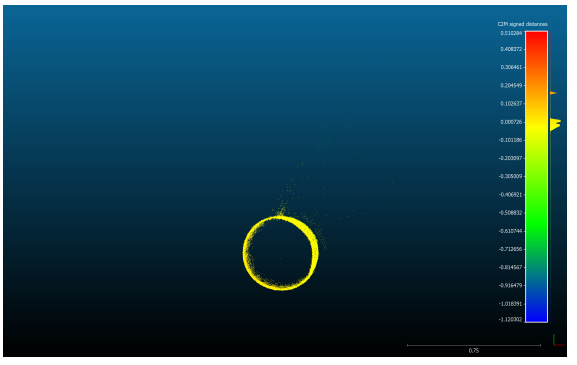

(c) Pico flexx

Figure 5. Cloud-to-mesh distances from the depth sensor to the 3D model (not shown). Screenshots from CloudCompare [78]. Please note that the color scale vary between the plots.

\subsection{Illumination Tests}

The sensors are tested in two test setups under a range of illumination levels. The setups are constructed as follows:

1. A university laboratory containing no windows in order to emulate the complete darkness of certain sections of a sewer pipe. A standard $\varnothing 400 \mathrm{~mm}$ PVC pipe with a length of $2 \mathrm{~m}$ is located on top of a metal frame that enables easy access for scientific experimentation. The setup is shown in Figure 6.

2. An above-ground outdoor test setup consisting of four wells connected by Ø200 mm and $\varnothing 400 \mathrm{~mm}$ PVC pipes. Each well may be covered by a wooden top to prevent direct sunlight from entering the pipe. The pipes are laid out in different configurations to simulate straight and curved pipes, branch pipes, and transitions between pipes of different materials and diameters. The pipes are covered with sand to block to block sunlight from entering the pipes and to enable rapid manipulation of the configuration of the pipes. The above-ground setup is pictured in Figure 7.

The depth sensors are tested through a wide variety of illumination levels enabled by adjusting the input voltage of the light sources. The intensity of the illumination is measured with a light meter [80] that is positioned with a distance of $40 \mathrm{~cm}$ from the 
light source. The laboratory experiments consist of 23 illuminance levels ranging from $0.02 \mathrm{~lx}$ to $294 \mathrm{~lx}$ whereas outdoor experiments consist of 9 illuminance levels ranging from $1.93 \mathrm{~lx}$ to $619 \mathrm{~lx}$. The finer granularity of the laboratory experiments enable the study of the importance of illumination to the depth sensors in finer detail while the higher illumination level from the outdoor experiments will reveal if the sensors benefit from even more light than present in the laboratory experiments. The details of the experimental setups are found in Table 3.

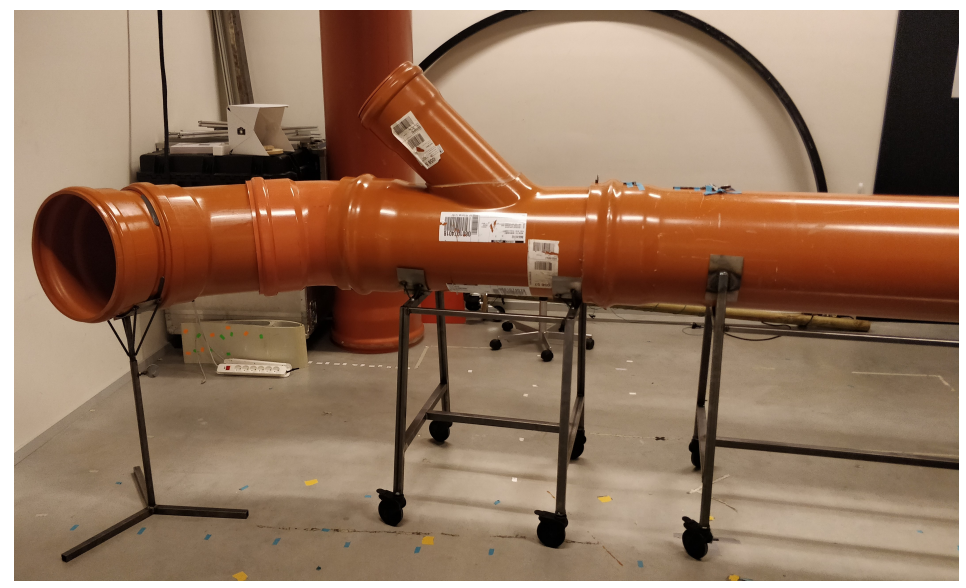

(a) View of the bigger setup consisting of a single branch pipe and bends.

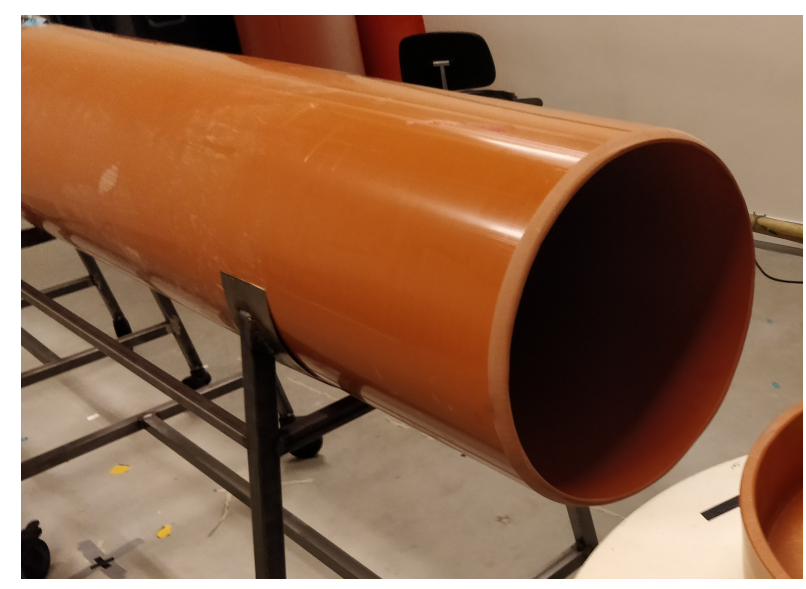

(b) Close-up view of the straight pipe used for testing.

Figure 6. Laboratory experimental setup.

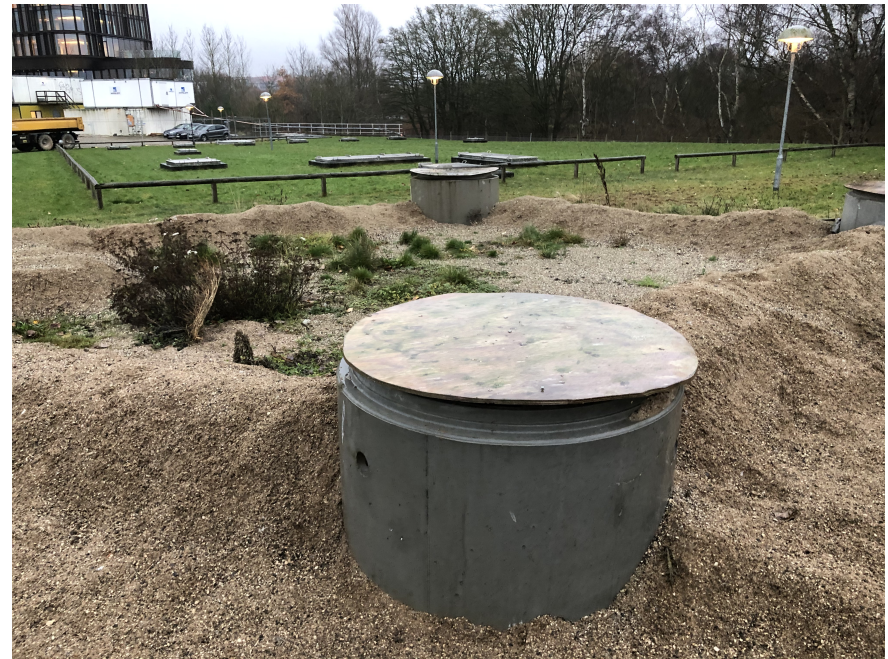

(a) View of two out of four wells of the outdoor setup. A glimpse of the third well is seen at the upper right corner of the image.

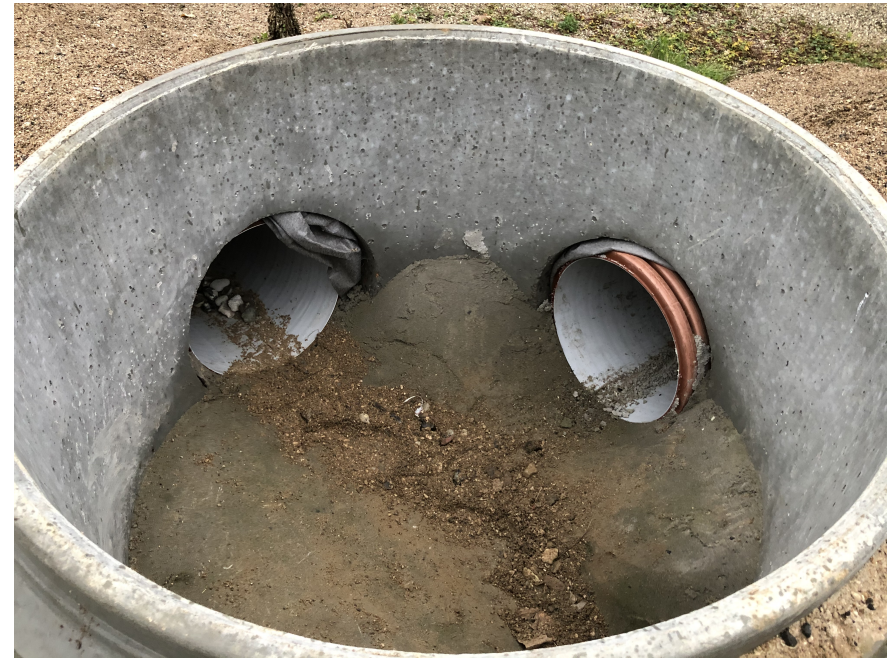

(b) Close-up view of the test well of the outdoor above-ground test setup.

Figure 7. Outdoor above-ground experimental setup. The four wells are laid out in a square configuration with $5 \mathrm{~m}$ between each corner.

Table 3. Configuration of the experimental setups for the illumination tests.

\begin{tabular}{lllllr}
\hline \multirow{2}{*}{ Test Setup } & \multirow{2}{*}{ Pipe } & \multirow{2}{*}{ Light Source } & \multicolumn{2}{c}{ Illuminance Levels } & Low \\
& & & High & \# Levels \\
\hline Lab & Ø400 PVC & Custom ring light & $0.021 \mathrm{~lx}$ & $294 \mathrm{~lx}$ & 23 \\
Outdoor & $\varnothing 400$ PVC & Smart Vision Lights S75-Whi [71] & $0.081 \mathrm{~lx}$ & $440 \mathrm{~lx}$ & 9 \\
\hline
\end{tabular}




\subsection{Water Level Tests}

The laboratory setup is reconfigured to allow for water to accumulate in the pipe. A piece of acrylic glass is inserted at both ends of the sewer pipe and fastened with glue as seen from Figure 8. The acrylic glass allows for water levels in the pipe between 0 and $80 \mathrm{~mm}$. The water level is measured as the distance between the surface of the water and the bottom of the pipe, illustrated in Figure 9. For the experiments, the water level is varied in steps of $20 \mathrm{~mm}$ from 0 to $80 \mathrm{~mm}$. In order to emulate the dirty water flowing through the real sewer pipes, we pollute clean tap water from the laboratory by adding sand, milk, and ground coffee. The small amount of sand models sediments in the pipes and the addition of milk and coffee renders the water opaque and cloudy. Details of the pollutants are found in Table 4. The reference 3D model described in Section 4.2 is modified to account for the different water levels of the experiments. For each water level experiment, a plane is injected into the $3 \mathrm{D}$ model that resembles the specific water line of the experiment. This construction entails that a point cloud from a sensor is not penalized if the point cloud contains points lying on the water line or contains points lying on the pipe floor below the water line.

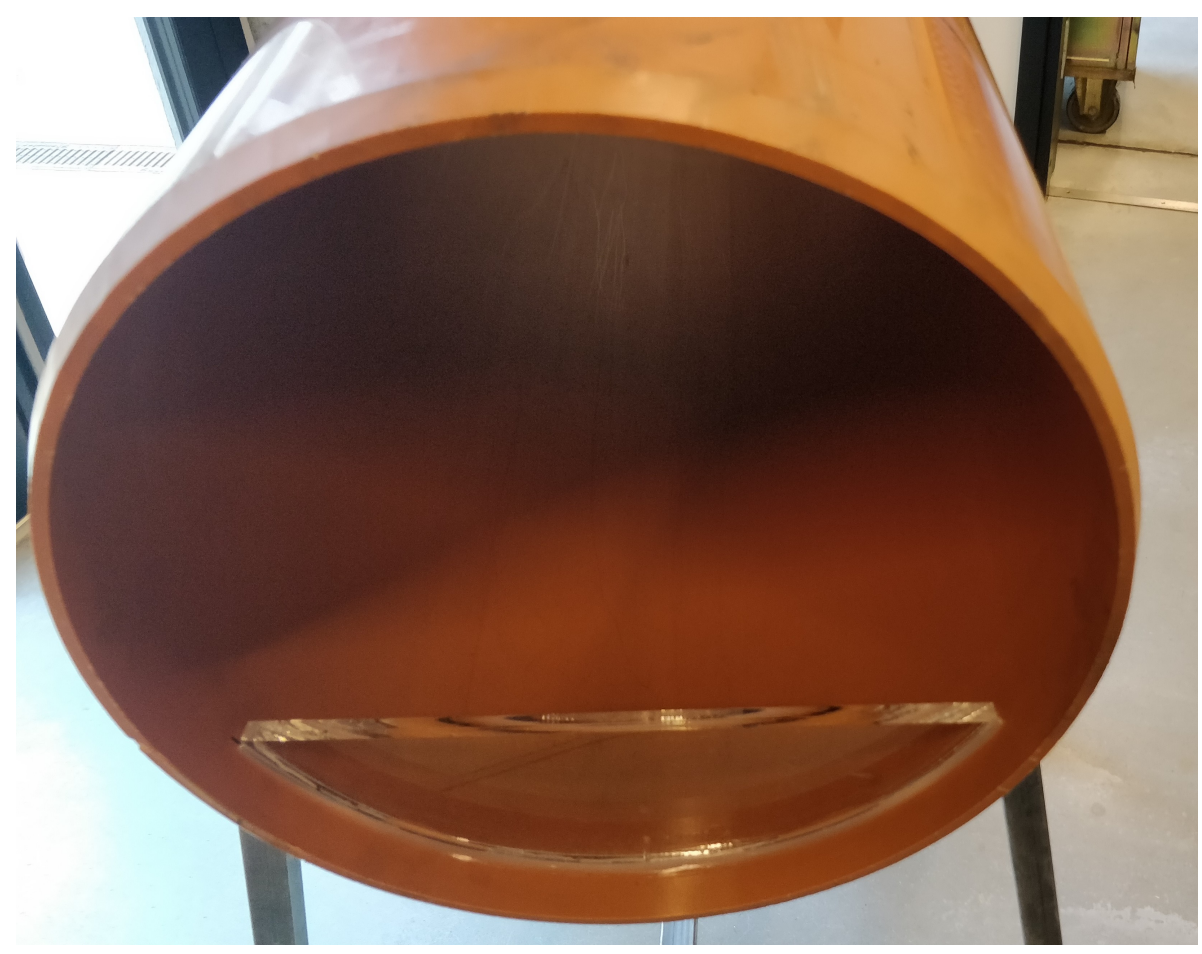

Figure 8. Water level setup. A piece of acrylic glass is inserted at both ends of the pipe in order to accommodate water. 


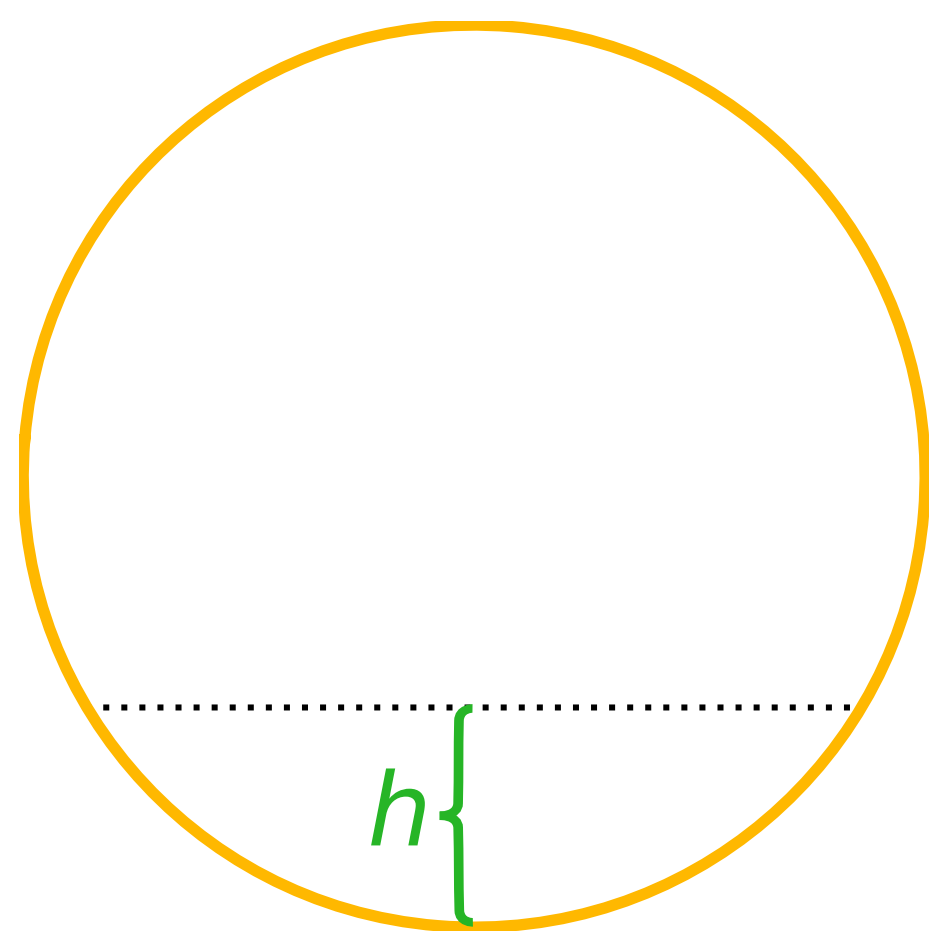

Figure 9. Cross-sectional view of the sewer pipe. The water level is measured by $h$ which is the line orthogonal to the water line and the bottom of the pipe.

Table 4. Details of the pollutants added to the laboratory water for the water level experiments. The amount of pollutants are found experimentally to maintain a certain level of cloudiness in the water.

\begin{tabular}{ccc}
\hline Water Level & Amount of Ground Coffee & Amount of Milk \\
\hline $0 \mathrm{~mm}$ & $0 \mathrm{~g}$ & $0.0 \%$ \\
$20 \mathrm{~mm}$ & $35 \mathrm{~g}$ & $0.5 \%$ \\
$40 \mathrm{~mm}$ & $97 \mathrm{~g}$ & $0.2 \%$ \\
$60 \mathrm{~mm}$ & $97 \mathrm{~g}$ & $0.5 \%$ \\
$80 \mathrm{~mm}$ & $270 \mathrm{~g}$ & $0.5 \%$ \\
\hline
\end{tabular}

\section{Experimental Results}

The results of the experimental tests of the illumination levels and water levels are described in the following.

\subsection{Illumination Tests}

The illumination tests are conducted by placing the LEGO-based robot inside the pipe such that the sensors and light source are placed at least $0.2 \mathrm{~m}$ inside the pipe. The placement of the robot inside the laboratory and outdoor pipes is seen in Figure 10. The input voltage to the light source is regulated in steps that adhere to the specifications from Table 3. After each adjustment of the input voltage, external light is blocked from the scene. In the laboratory setting, this is achieved by turning off the lights in the windowless room and in the outdoor setting, wooden covers are placed on top of the wells. The depth sensors are hereafter initialized and instructed to record footage of the scene for 5 to $10 \mathrm{~s}$. The sensor recordings are post-processed offline after the protocol described in Section 4.2. In step 3 of the post-processing, the point clouds are cropped such that $-2 \mathrm{~m} \leq x \leq 2 \mathrm{~m}$, $-2 \mathrm{~m} \leq y \leq 2 \mathrm{~m}$, and $0 \mathrm{~m} \leq z \leq 2.5 \mathrm{~m}$. The $x$ and $y$ range is deliberately chosen to be very permissive such that a robot would be able to drive in very large pipes without any assumptions. The $\mathrm{z}$ range is chosen due to the maximum extent of the investigated pipes. 


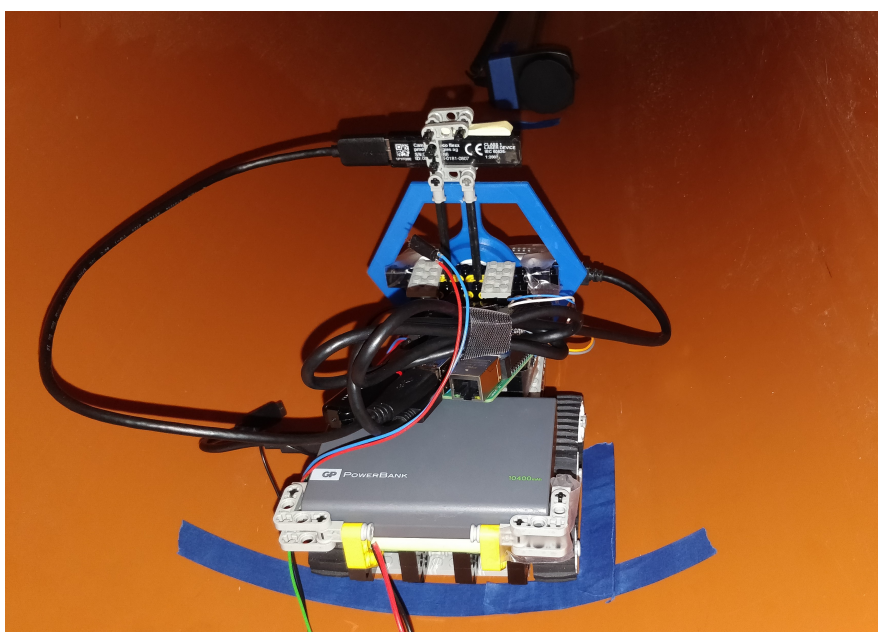

(a) Laboratory

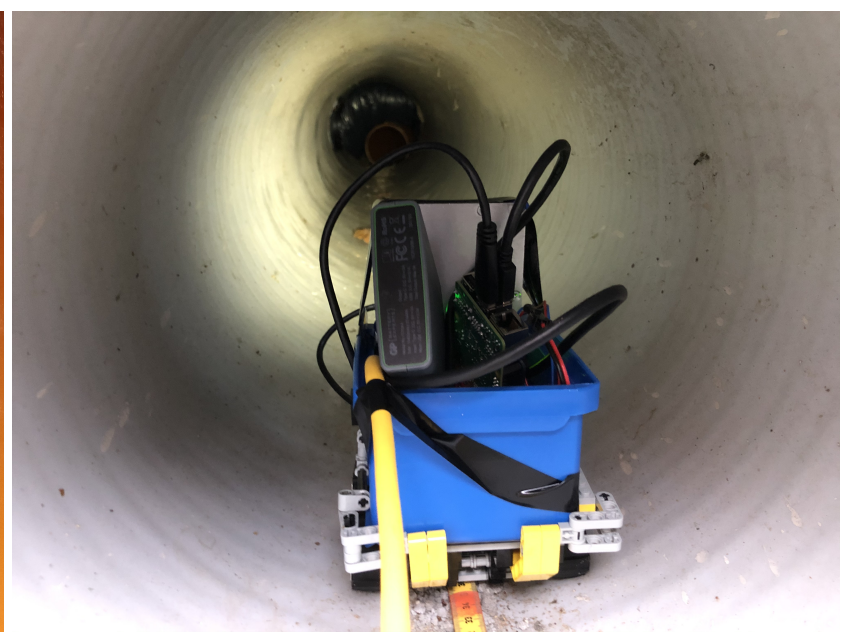

(b) Outdoor

Figure 10. View of the LEGO-based robot located in the laboratory (a) and outdoor (b) pipes. The lux meter is visible in the top of image (a). The lux meter was not present during the recording of sensor data.

The aggregated results of the overall reconstruction performance with respect to the amount of light available in the pipes are shown in Figure 11. From the first glance at the results from Figure 11, the pico flexx camera seems superior to both Realsense configurations in terms of both mean average error and standard deviation. The performance of the pico flexx is, as expected, not affected by the absence or presence of visual light in the pipes.

This is a different story for the Realsense cameras. In the following, we will use the following short names for the Realsense: Realsense-Off for the Realsense camera with the infrared emitter turned off and Realsense-On for the Realsense camera with the infrared emitter turned on. Under low-light conditions, the Realsense-On camera is the better Realsense camera. Surprisingly, however, is the observation that the reconstruction performance of the Realsense-On camera does not improve with the addition of more light in the scene. On the contrary, the mean reconstruction error seems to slightly increase with the addition of more light. This suggests that the Realsense-On camera almost entirely relies on the reflections from the infrared dot projector.

On the other hand, the Realsense-Off camera benefits immensely from the presence of infrared and visual light. This is expected, as the camera in this configuration is a passive stereo camera that relies entirely on the presence of visual light. This is evident from Figure 11 which shows a mean reconstruction error between $0.085 \mathrm{~m}$ and $0.105 \mathrm{~m}$ in the presence of virtually no visible light $(0.02 \mathrm{~lx}$ and $1.93 \mathrm{~lx})$. The results show that the Realsense-Off camera is the better Realsense camera when enough light is present in the scene. In the laboratory experiments, the threshold for "enough light" is at $51 \mathrm{~lx}$, and in the outdoor experiments, the threshold is found at $221 \mathrm{~lx}$.

As evidenced by the plots for the mean and standard deviation, there is a significant reduction in standard deviation of the reconstruction error by the Realsense-Off camera when more light is present in the scene. The reduction is most pronounced in the laboratory setting with a decrease of $54 \%$ between 0.22 lx and $294.11 x$ whereas in the outdoor setting, the reduction is $34 \%$ between $0.00 \mathrm{~lx}$ and $286.3 \mathrm{~lx}$.

The bottom of Figure 11 shows the average number of valid depth points per depth frame from the three camera configurations. The plots support the observation that the pico flexx camera and the Realsense-On camera are insensitive to the presence of light. In the outdoor scenario, the pico flexx camera produces on average 34,900 valid depth points per frame whereas the Realsense-On camera 424,000 points per frame. The Realsense-Off camera produces 59,000 points at 0.00 lx which monotonically increase to 139,100 points at $286 \mathrm{~lx}$ and 206,150 points at $465 \mathrm{~lx}$. 

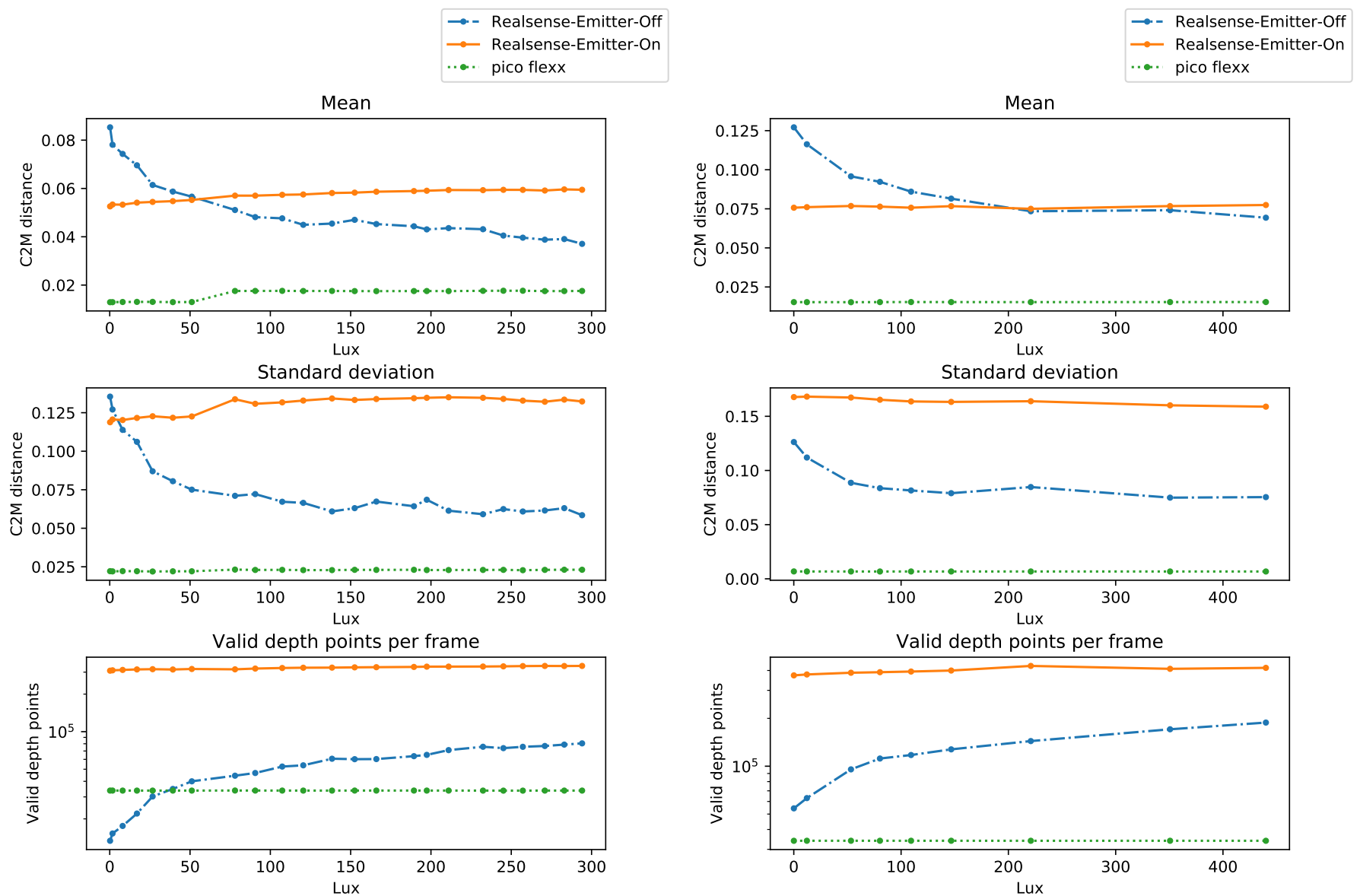

(a) Laboratory

(b) Outdoor

Figure 11. Mean (top), standard deviation (middle) of the reconstruction error, and (bottom), average number of valid depth points from each depth image of the camera. C2M distance: cloud to mesh distance.

Looking at Figure 11a, the Realsense-Off camera produces significantly fewer valid depth points in the laboratory setting than in the outdoor setting; 13,350 points at $0.22 \mathrm{~lx}$ and 80,580 points at $294 \mathrm{~lx}$ in the laboratory experiments, a decrease of $77 \%$ and $42 \%$ compared to the respective illumination levels in the outdoor setting. The differences may have many causes, including the change of light source between the experiments. Another cause for the change might be the difference in materials of the interior of the sewer pipes $\mathrm{z}$ is noticeable in Figure 10. The pipe for the laboratory experiments consists of only one type of plastic which features a highly reflective surface whereas the interior of the outdoor pipe contains a white plastic lining with a less reflective coating. Estimation of corresponding points with a passive stereo camera is significantly harder on reflective surfaces and might thus explain some of the variation between the experiments.

\subsubsection{Reconstruction of the Cylindrical Pipe}

The above results show the average reconstruction error with respect to the amount of light available in the scene. The following analysis will investigate how the reconstruction error is distributed throughout the extent of the cylindrical pipe. In order to show the reconstruction error of the three-dimensional pipe on two-dimensional paper, the cylinder is unfolded to the two-dimensional plane by the following equations:

$$
\begin{aligned}
& y_{2 \mathrm{D}}=\theta=\operatorname{atan} 2\left(x_{3 \mathrm{D}}, y_{3 \mathrm{D}}\right) \\
& x_{2 \mathrm{D}}=z_{3 \mathrm{D}}
\end{aligned}
$$


where $x_{2 \mathrm{D}}, y_{2 \mathrm{D}}$ denote the $x$ and $y$ coordinates, respectively, of the two-dimensional plane and $x_{3 \mathrm{D}}, y_{3 \mathrm{D}}$, and $z_{3 \mathrm{D}}$ denote the $x, y$, and $z$, coordinates, respectively, of the threedimensional point cloud.

The mapping might be visualized by imagining a cylinder which is cut at the top $(x=0 \mathrm{~m}, y=r)$ along the $z$-axis where $r$ denotes the radius of the pipe. The cylinder is then unfolded to lie in the $y z$-plane. Normal convention would compute $\theta=\operatorname{atan} 2(y, x)$. However, it is deliberately chosen to compute $\theta=\operatorname{atan} 2(x, y)$ such that the origin $(\theta=0)$ is at the top of the cylinder at $x=0 \mathrm{~m}, y=r$. As a result of the mapping, points that do not lie directly on the cylinder are projected to the closest point on the cylindrical surface by a line that is orthogonal to the $z$-axis. The mapping is visualized in Figure 12.

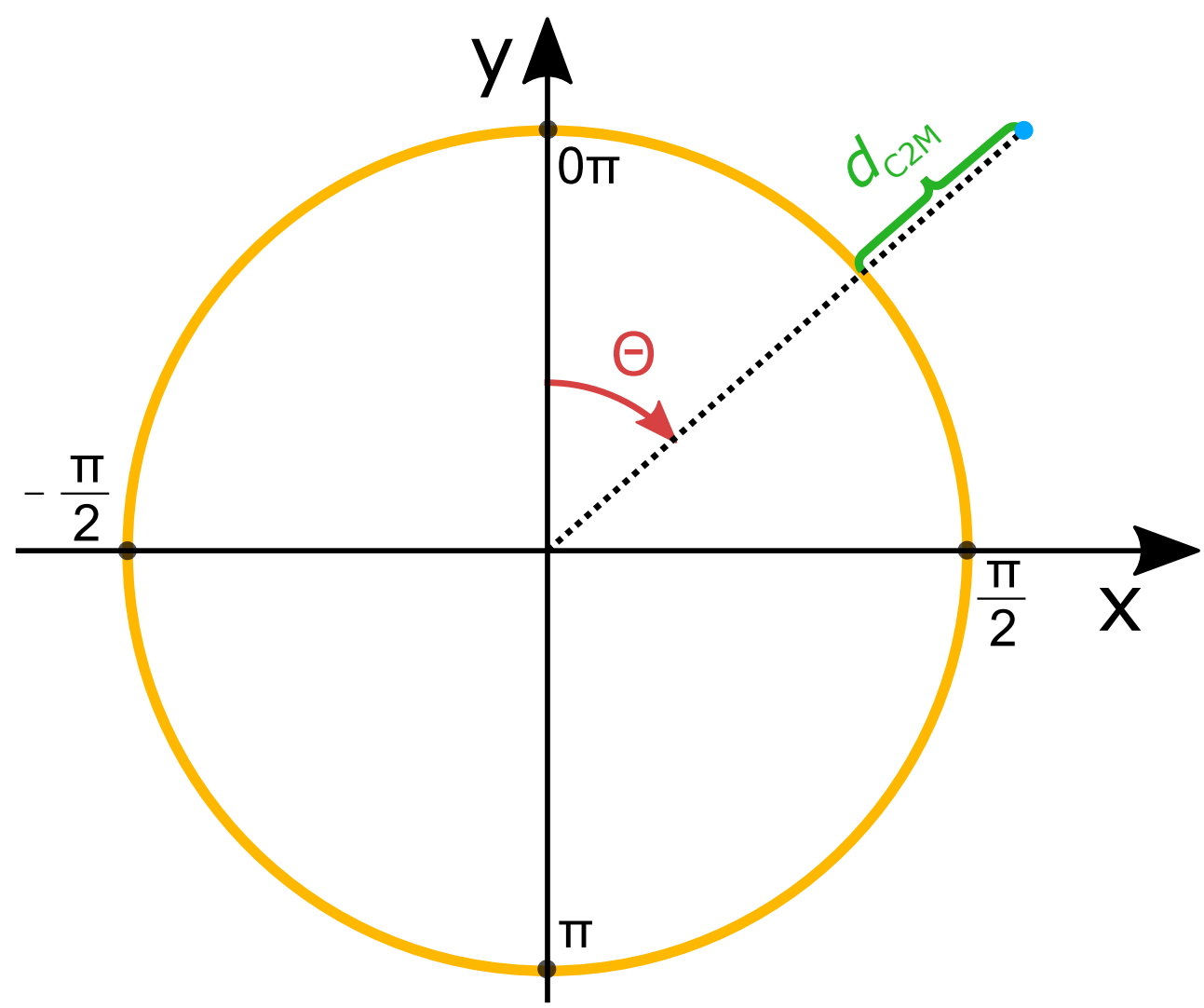

Figure 12. Illustration of the $3 \mathrm{D}$ to $2 \mathrm{D}$ mapping of the cylinder. The cross section of the cylinder is seen from the $X Y$-plane. The angle $\theta$ is mapped onto the $y$-axis of the two-dimensional plots.

The mapping of the cloud-to-mesh reconstruction error from the laboratory and outdoor experiments are shown in Figures 13 and 14, respectively. The figures show the reconstruction of a single depth frame for a range of illuminance levels. The depth frames are not cherry-picked but programmatically selected from a range of frames after an initial warm-up of the depth sensors. As seen from the mean reconstruction error and the number of depth points from Figure 11, the Realsense-Off camera is the only camera configuration to be significantly affected by the absence or presence of light. For this reason, a range of four illuminance levels is visualized for the Realsense-Off camera and only two illuminance levels for the Realsense-On and pico flexx cameras, respectively. 

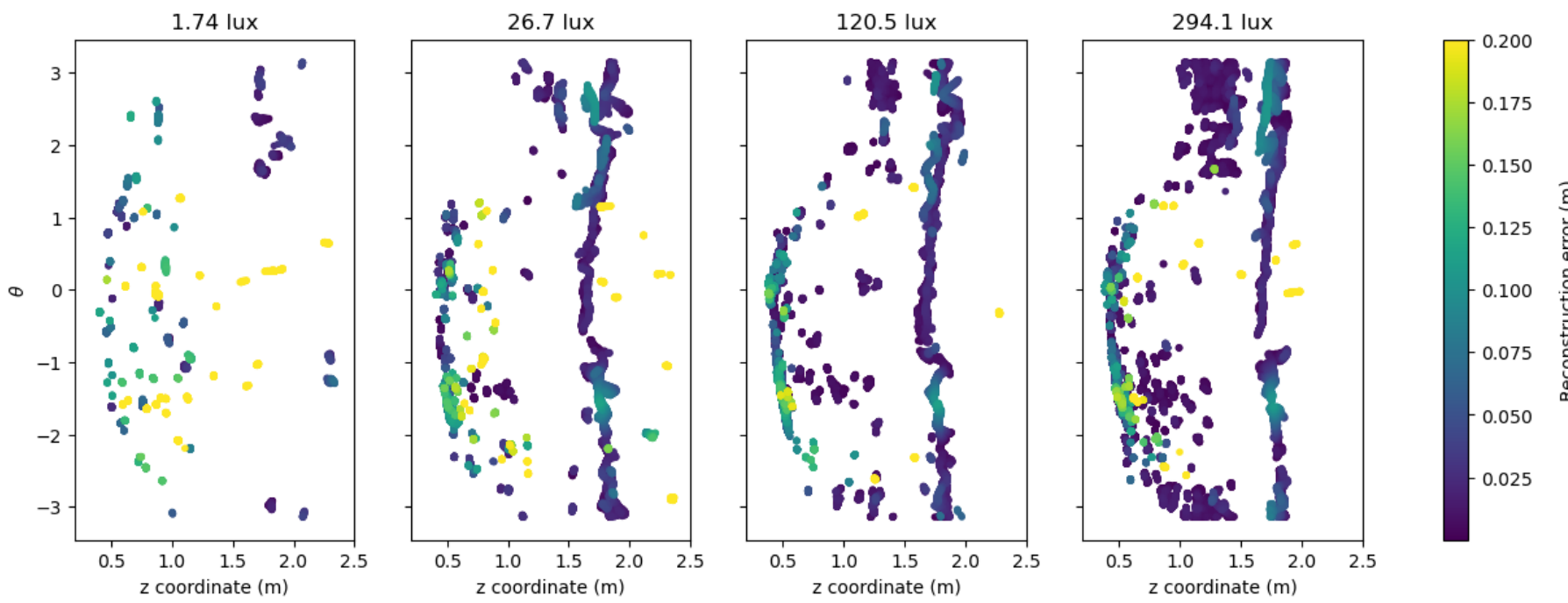

(a) Realsense Emitter Off

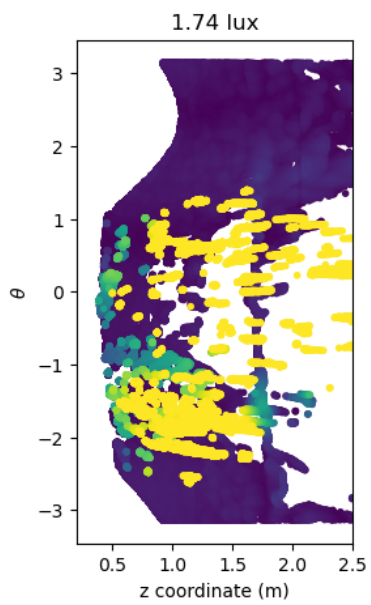

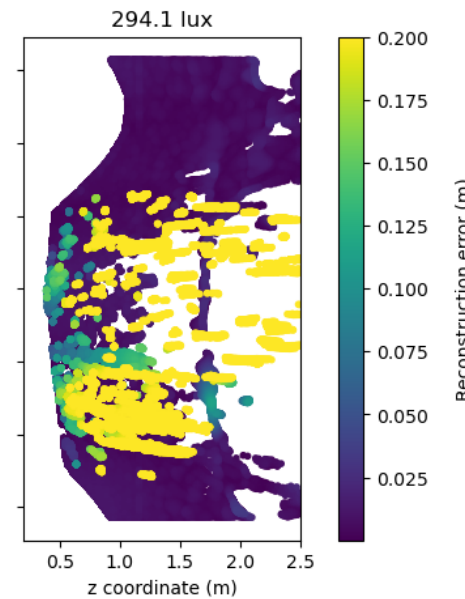

(b) Realsense Emitter On
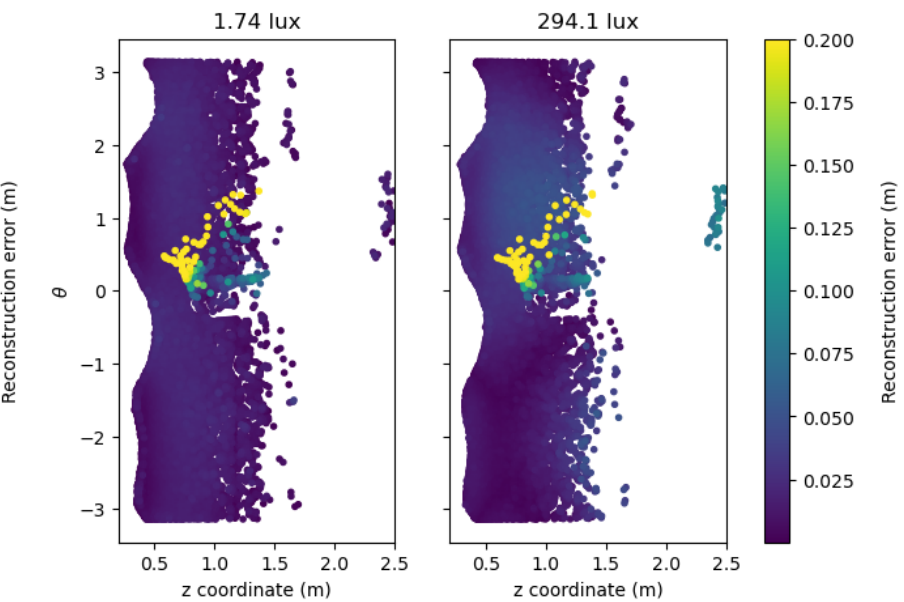

(c) Pico flexx

Figure 13. 2D projection of the cloud-to-mesh distance from the three sensor configurations in the laboratory setup. The color coding of the cloud represents the cloud-to-mesh distance. If points overlay each other on the 2D projection, the point with the largest cloud-to-mesh distance is plotted on top and is thus fully visible.

A glance at Figures 13a and 14a reveals a low, unevenly distributed point cloud of the Realsense-Off camera, which renders these point clouds useless for 3D reconstruction for robotic applications. According to Figure 11, the Realsense-Off camera produces more points for illuminance levels above $50 \mathrm{~lx}$ than the pico flexx camera, but the points from the Realsense-Off camera are not evenly distributed throughout the pipe. When looking at the reconstructions from Figures 13a and 14a, most of the depth points seem to be located in bands either along the $z$-axis (laboratory) or along curved segments constrained along the $\theta$-axis (outdoor). 

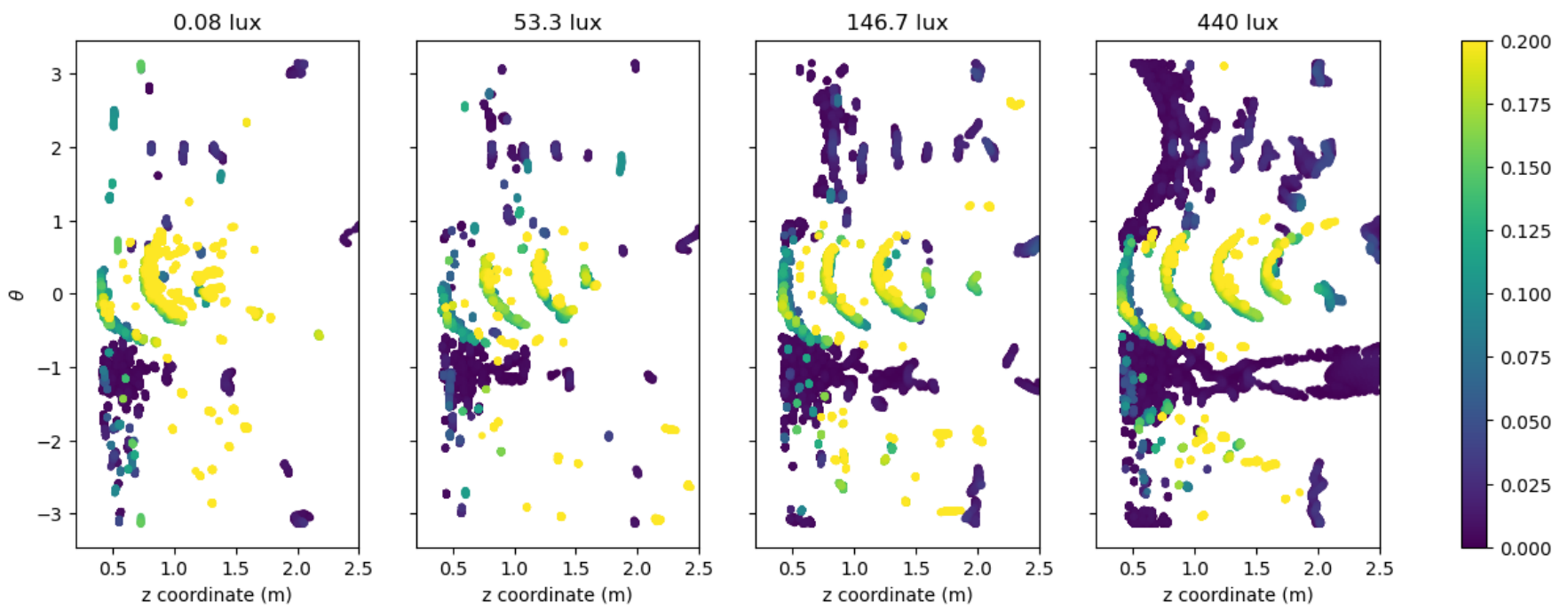

(a) Realsense Emitter Off
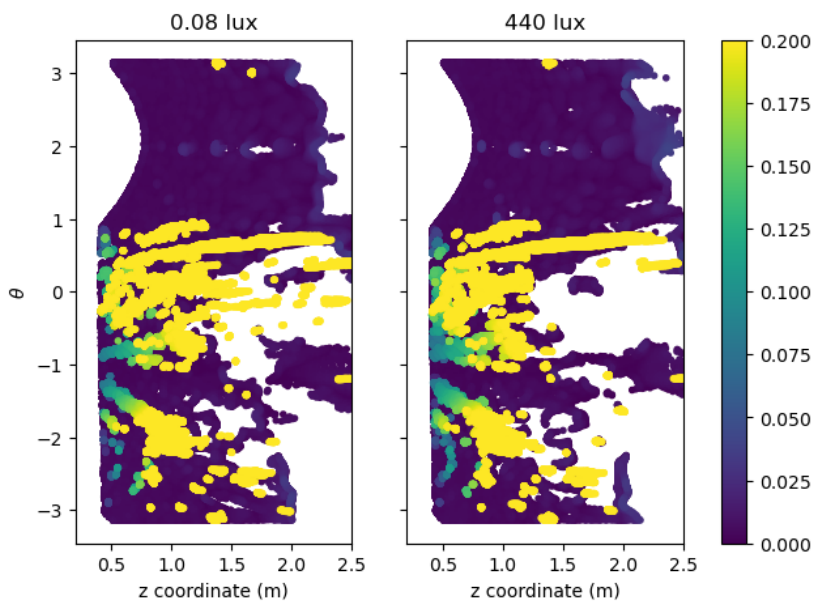

(b) Realsense Emitter On

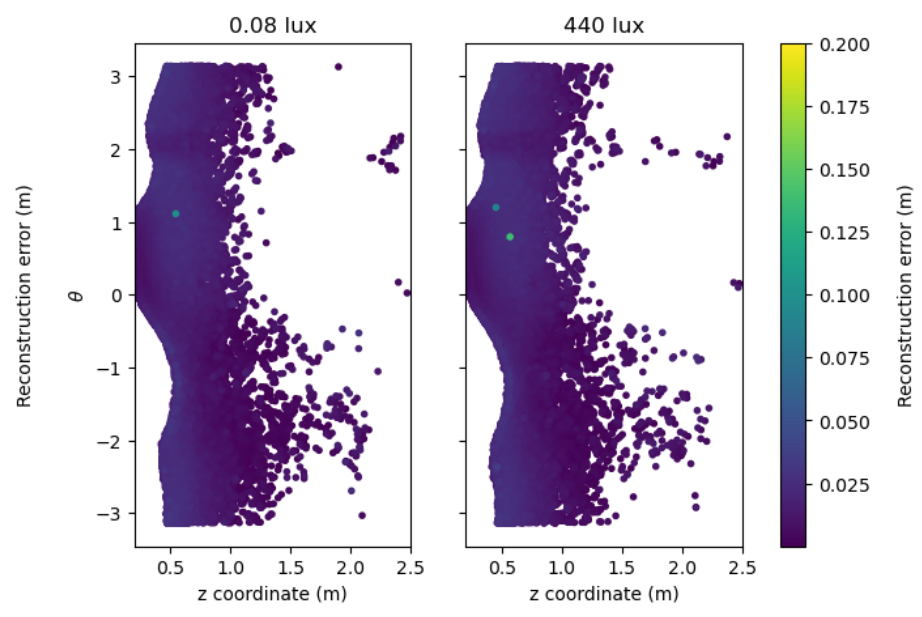

(c) Pico flexx

Figure 14. 2D projection of the cloud-to-mesh distance from the three sensor configurations in the outdoor setup. The color coding of the cloud represents the cloud-to-mesh distance. If points overlay each other on the 2D projection, the point with the largest cloud-to-mesh distance is plotted on top and is thus fully visible.

The addition of the infrared emitter radically changes the output from the Realsense camera, producing a dense, smooth cloud of the pipe. This is apparent from the dense dark blue areas on Figures $13 \mathrm{~b}$ and $14 \mathrm{~b}$. The $z$-range of the cloud is longer at the bottom of the pipe $(\approx 2 \mathrm{~m})$ and shorter at the top of the pipe $(\approx 1-5 \mathrm{~m})$. However, the smooth cloud is ridden from a large number of outliers apparent as yellow sections on the 2D projections. Their errors extend far beyond the threshold on $0.2 \mathrm{~m}$ of the visualization and contribute to the large average error and standard deviation reported in Figure 11. These outliers are hard to filter out as they are placed randomly throughout the 3D space. Without the cropping operation performed in the post-processing step, the number of outliers would be even larger. Filtering the outliers from the Realsense-On camera is a computationally heavy operation due to the large amount of points and requires knowledge of the geometry of the pipe in order to succeed.

The point cloud from the pico flexx camera provides a consistent reconstruction with few outliers. Compared with the "good" sections of the reconstruction from the RealsenseOn camera, the pico flexx point cloud shows a slightly higher error but without the many outliers of the former camera. The point cloud on the reflective laboratory pipe contains more outliers than on the matt surface of the outdoor pipe. However, the point cloud 
on the laboratory pipe is denser than its outdoor counterpart within the range of $0.5 \mathrm{~m}$ to $1.5 \mathrm{~m}$.

\subsubsection{Reconstruction Performance Along the z-axis}

The performance of the point cloud may also be studied by investigating the point density and reconstruction error as a function of the depth of the pipe, e.g., the $\mathrm{z}$-coordinate of the measurements. The average performance throughout all illuminance levels is shown in Figure 15.

The results from Figure 15 support the conclusions from Section 5.1.1. The pico flexx camera features a higher point density in the laboratory pipe within a range of $0.5 \mathrm{~m}$ to $1.5 \mathrm{~m}$ when compared with the outdoor pipe. However, the amount of points in the cloud above $1.5 \mathrm{~m}$ drops sharply in the reflective laboratory pipe whereas it gradually decreases in the outdoor pipe. At $1.5 \mathrm{~m}$, however, the amount of points from the pico flexx camera drops below 10 points $/ \mathrm{mm}$ along the $z$-axis which indicates that reconstruction beyond this depth is increasingly difficult. Looking at the reconstruction error of the pico flexx, it is observed that the camera produces a more accurate point cloud from the matt outdoor pipe than the reflective outdoor pipe. At $0.5 \mathrm{~m}$, the mean reconstruction error lies at $\approx 0.02 \mathrm{~m}$ for both pipes. Above $0.5 \mathrm{~m}$, the error increases slightly in the laboratory pipe but decreases in the outdoor pipe.

The dense point cloud from the Realsense-On camera starts at distance of $0.44 \mathrm{~m}$ with $20,000-66,000$ points per mm which gradually decreases to $650-900$ points per $\mathrm{mm}$ at the cut-off depth at $2.5 \mathrm{~m}$. The graceful decrease in point density along the $z$-axis is a virtue that the active depth sensor shares with the pico flexx. The mean reconstruction error of the Realsense-On camera, however, is heavily impaired by the many outliers and does not seem to converge to a certain trend as the distance to the sensor increases.

This is also the case for the Realsense-Off camera. The point cloud from the camera provides fewer outliers than the Realsense-On camera, but the overall point cloud is so sparse that the "good" points cannot correct for the error of the outliers. The point density of the Realsense-Off camera is generally below the Realsense-On camera. In certain sections on the $z$-axis, however, the point density touches the Realsense-On camera and drops hereafter.
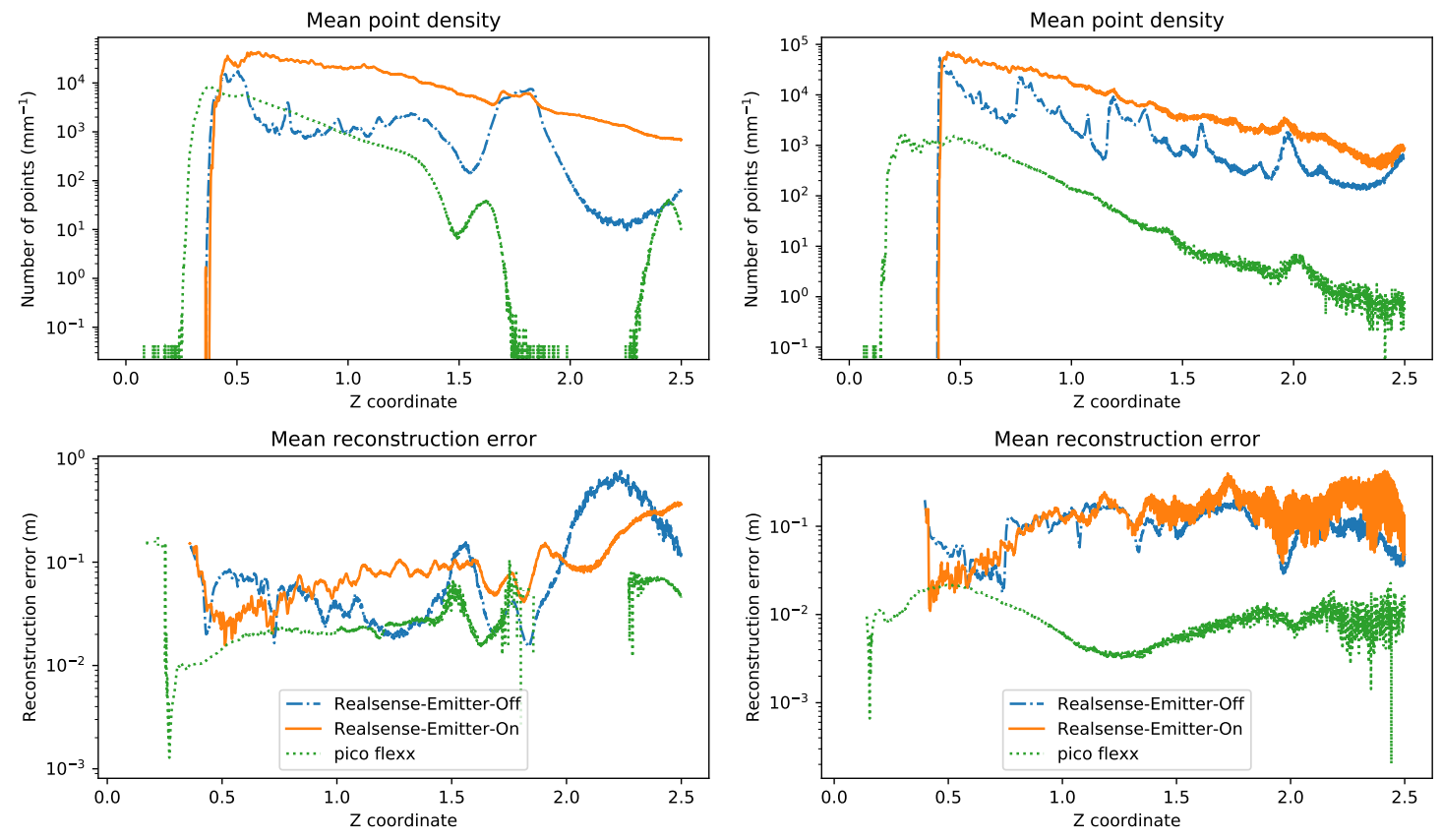

(a) Laboratory

(b) Outdoor

Figure 15. Mean point density and reconstruction error (cloud-to-mesh distance) with respect to the depth (z) coordinate of the measurement. 


\subsection{Water Level Tests}

The water level tests are conducted by placing the robot just above the acrylic cover of the laboratory setup described in Section 4.4. As described in Table 4, the water level is adjusted from $0 \mathrm{~mm}$ to $80 \mathrm{~mm}$ in steps of $20 \mathrm{~mm}$. The water level tests are performed with the Realsense-On and pico flexx cameras, and the post-processing of the sensor data is performed according to the instructions of Section 4.2. The point clouds are cropped such that $-2 \mathrm{~m} \leq x \leq 2 \mathrm{~m},-2 \mathrm{~m} \leq y \leq 2 \mathrm{~m}$, and $0 \mathrm{~m} \leq z \leq 1 \mathrm{~m}$. The $z$ range is limited as a consequence of the short length of the pipe used for the water level experiments.

The reconstruction error of the point clouds is visualized by unfolding the cylindrical pipe as described in Section 5.1.1 and by showing the point cloud from the XY-plane. The reconstructions from the Realsense-On and pico flexx cameras are shown in Figures 16 and 17, respectively. The water level and the geometry of the cylindrical pipe are overlaid as orange lines and orange circles, respectively, to allow for easier interpretation.
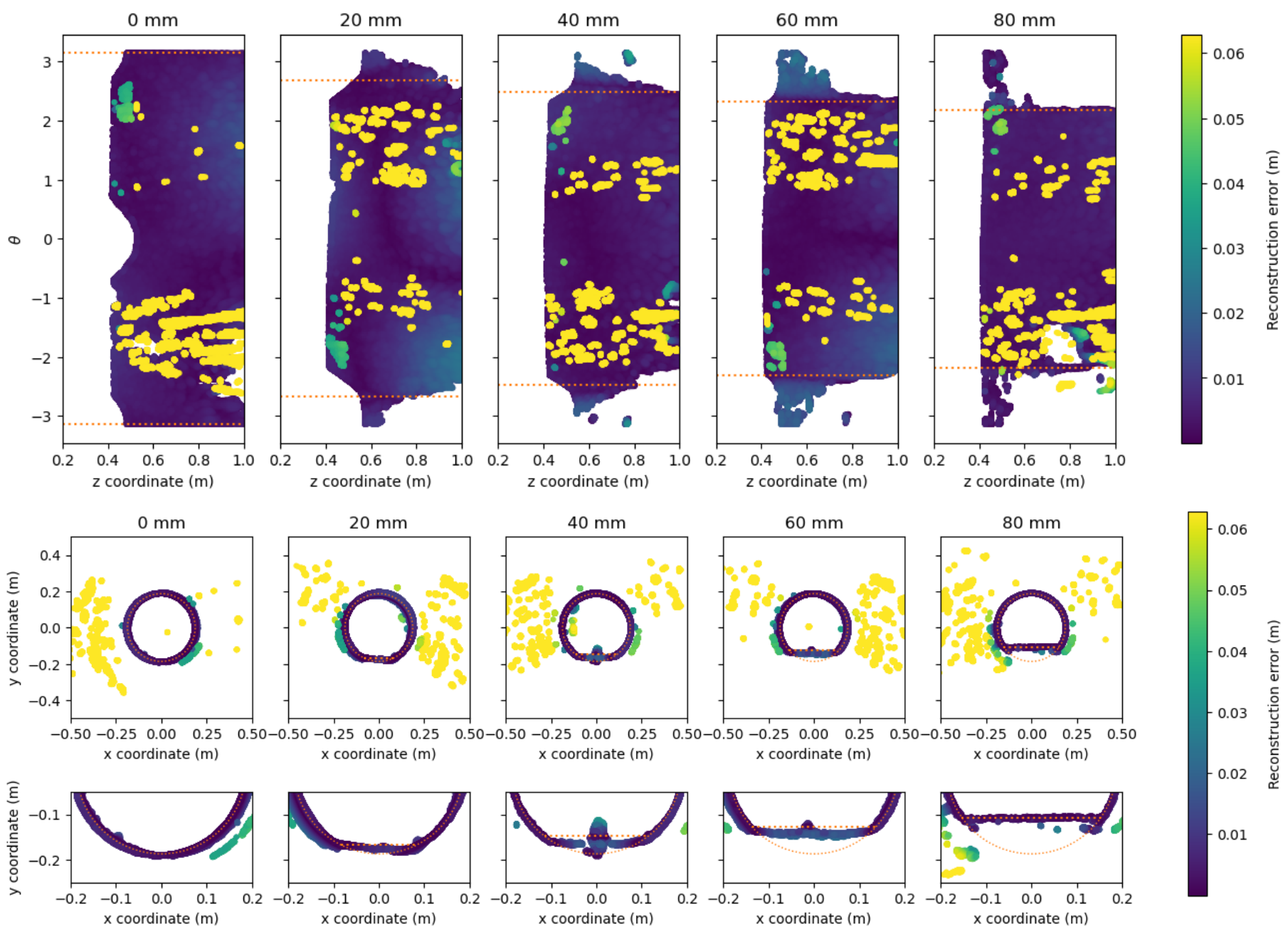

0.01

Figure 16. Reconstructed point cloud for the water level experiments by the Realsense-On camera. The water level and pipe geometry is indicated with the orange line. The points are color-coded according to the cloud-to-mesh distance to the reference pipe. (Top) unfolded view of the reconstructed pipe. The water level is indicated with an orange dotted line. (Middle) View from the XY-plane of the reconstructed pipe. (Bottom) Zoomed in view of the XY-plane of the reconstructed pipe. 

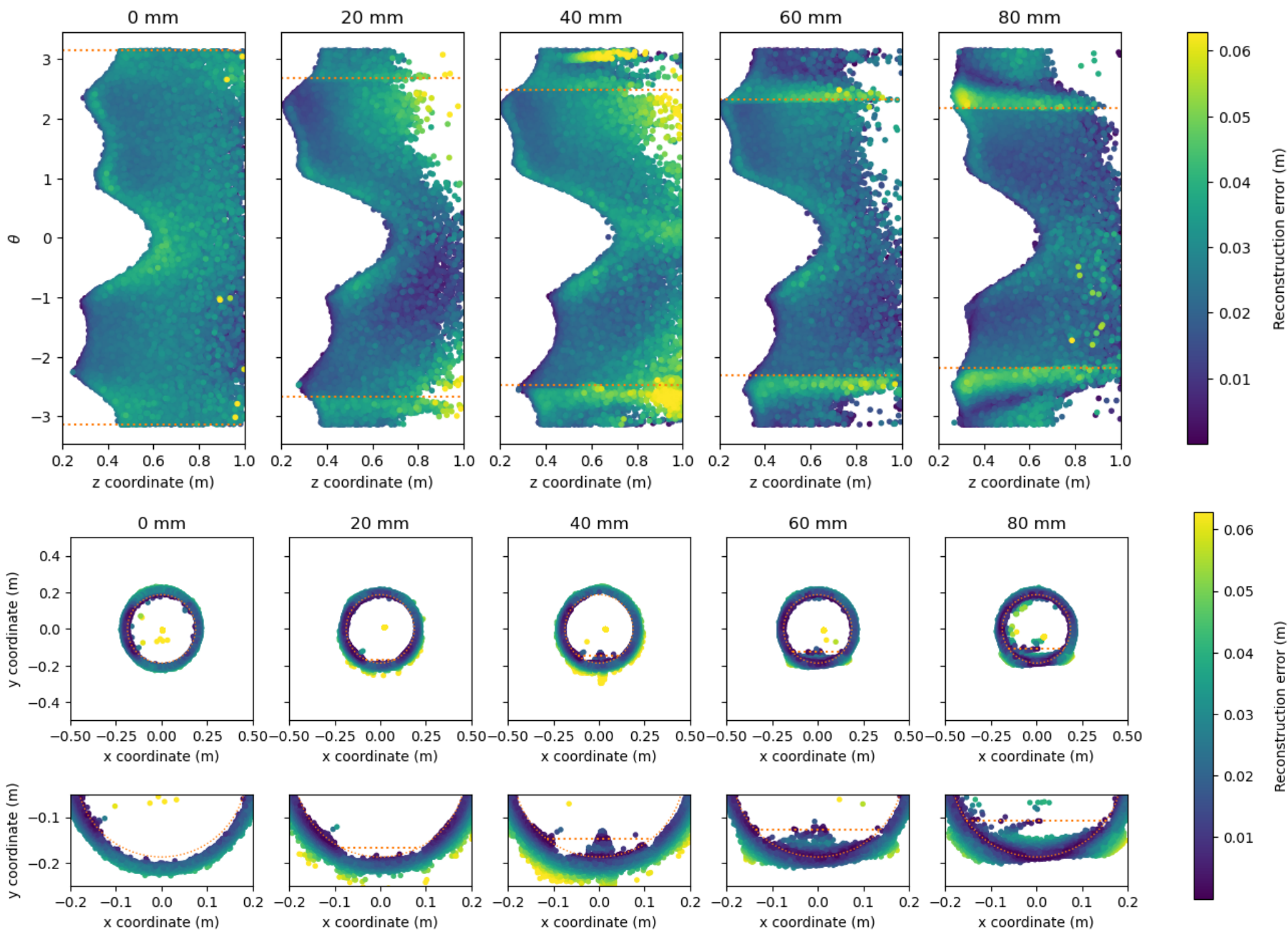

Figure 17. Reconstructed point cloud for the water level experiments by the pico flexx camera. The water level and pipe geometry is indicated with the orange line. The points are color-coded according to the cloud-to-mesh distance to the reference pipe. (Top) unfolded view of the reconstructed pipe. The water level is indicated with an orange dotted line. (Middle) View from the XY-plane of the reconstructed pipe. (Bottom) Zoomed in view of the XY-plane of the reconstructed pipe.

The point cloud from the Realsense-On camera approximately follows the water line, i.e., it is possible to estimate the water level by observing the lack of points where water is present in the pipe. This is evident by studying the unfolded view of the pipe and the close-up view of the XY-plane visualized in Figure 16. The dense cloud of inlier points that is close to the reference pipe is hampered by the strong presence of outliers that goes far beyond the threshold of the color scale of the cloud-to-mesh distance. The spatial extent of the outliers is noticeable from the top and middle plots of Figure 16. Consistent with the results from the clean pipes, the outliers from the Realsense-On camera are scattered randomly in 3D-space, leaving it hard to filter the point cloud to recover the actual pipe.

As opposed to the Realsense-On camera, the pico flexx camera provides a significant amount of points below the water level, rendering it difficult to estimate the water level from the cloud. When inspecting the point cloud at water levels between $40 \mathrm{~mm}$ to $80 \mathrm{~mm}$ from Figure 17, it is apparent that the cloud-to-mesh error increases slightly below the water level. The increase is seen in the bottom of Figure 17 as a slight bulge in the bottom of the point cloud. The point cloud that represents the remainder of the pipe has few outliers. When inspecting the inlier points of the cloud, the green shades of the cloud reveal that the cloud is less accurate than the inlier points of the Realsense-On camera. The slightly 
inaccurate cloud, however, might be a good trade-off for the sharp decrease in outliers in the water-less parts of the pipe compared with the Realsense-On camera.

\section{Conclusions}

We have compared the 3D reconstruction capabilities within the domain of sewer inspection of three different depth sensing technologies: passive stereo, active stereo, and ToF. The Realsense D435 camera is chosen as a representative of both the passive stereo and active stereo techniques as the camera is equipped with an infrared emitter that may be turned on an off; producing an active and a stereo camera, respectively. The PMD CamBoard pico flexx is chosen as a small, low-power ToF camera.

In order to compare the sensors, a laboratory and an above-ground outdoor setup were created to model confined sewer environments. The laboratory setup consists of a reflective $\varnothing 400 \mathrm{~mm}$ straight plastic pipe whereas the above-ground setup consists of four wells connected by $\varnothing 200 \mathrm{~mm}$ and $\varnothing 400 \mathrm{~mm}$ pipes which are used for our experiments. We have tested the capabilities of the depth sensors to reconstruct the pipes under a range of different illuminance levels; from virtually no light to $294 \mathrm{~lx}$ and $440 \mathrm{~lx}$ for the laboratory and outdoor setups, respectively. Furthermore, we have tested the performance of the ToF and active depth sensors under the presence of water in the pipe. The laboratory setup is used to simulate four different water levels, from $20 \mathrm{~mm}$ to $80 \mathrm{~mm}$ of water measured from the bottom of the pipe.

The point clouds acquired from the depth sensors are compared with 3D reference models of the pipes. The cloud-to-mesh distance between the point cloud and the 3D reference mesh is used as the main metric for assessing the reconstruction capabilities of the sensors.

The illumination tests show that both the pico flexx camera and Realsense camera with the emitter turned on (Realsense-On) are not affected by the presence nor absence of visual light. This was expected for the ToF pico flexx camera but not for the active stereo RealsenseOn camera. As expected, the presence of light is instrumental for the Realsense camera with the emitter turned off (Realsense-Off). For the Realsense-Off camera, there is a clear correlation between the increase of light available in the scene and the decrease in cloudto-mesh distance of the acquired point cloud from the sensor. Surprisingly, the RealsenseOff camera outperforms the Realsense-On counterpart when the scene is sufficiently lit. The reason for this is found in the large amount of outliers produced by the RealsenseOn camera that break the otherwise dense and accurate reconstruction produced from said camera. Even though the point cloud from the Realsense-Off camera is short of the outliers that haunt the Realsense-On camera, the point cloud from the camera is sparse and unevenly distributed. This implies that the point clouds from both Realsense configurations are not suited for the purpose of 3D reconstruction in a sewer inspection context.

Compared with the Realsense-On camera, the point cloud from the pico flexx camera contains significantly fewer points than the Realsense-On camera, and in the chosen camera configuration, the amount of points beyond a distance of $1.5 \mathrm{~m}$ are insignificant. However, the point cloud contains few outliers and gives an accurate, dense reconstruction of the sewer pipe within a range of 0.5 to $1.5 \mathrm{~m}$.

The water level tests show the same pattern with respect to the sensors; the point cloud from the Realsense-On camera is dense but noisy, and the point cloud from the pico flexx camera is less dense but more accurate. Furthermore, the tests reveal that the sensors observe the water level differently; the Realsense-On camera does not produce depth points on the water line whereas the pico flexx camera produces less accurate depth points that resembles the sections of the sewer pipe below the water line.

Overall, the comprehensive analysis of the results from the illumination tests and water level tests shows that the ToF pico flexx camera is superior to the passive and active Realsense configurations. The detrimental performance of the Realsense cameras might be caused by the reflective, featureless surfaces of the tested sewer pipes that impede the stereo matching process. However, the consistently high amount of outliers from the 
Realsense-On camera is a surprise. Future work could investigate if this is isolated to the specific Realsense sensor or is an issue with active stereo sensors in general. In order to utilize the data from the Realsense camera, an autonomous system must invest in heavy online post-processing of the acquired point clouds and impose strict constraints on the structure of the data, for instance, on the cylindrical shape of the pipe.

The pico flexx camera provides a compact, low-power device for depth sensing. While the spatial resolution of the depth image is significantly lower than the Realsense competitor, the camera makes up for this by providing an accurate point cloud that is unaffected by the presence or absence of light in the pipe. Overall, the pico flexx camera may be viewed as a favorite for an autonomous inspection system as the high accuracy of the point cloud reduces the need for expensive, on-board post-processing.

In this study, we have chosen to focus on depth sensing technology that provides readily available depth data with low post-processing requirements. However, one should not underestimate the importance of visual data for the purpose of detection of defects, assessment of structure, and offline validation of the system by humans. As evidenced in the literature review, there is a large body of work that focuses solely on the reconstruction of pipes from monocular and stereo imagery. The investigated depth sensors could easily be integrated with a stereo matching approach. A combined solution could offer a solution for real-time depth data and offline, detailed 3D reconstruction.

Previous works consider offline post-processing of the recorded data in order to reconstruct the pipe. If only relying on post-processing, this assumes that a human operator is able to guide the inspection vehicle as it traverses the pipe. With the exception of LiDAR and laser profilometry methods, stereo matching techniques are computationally expensive and not something that can be accommodated on a space and energy constrained inspection vehicle.

The current work investigates how recent advances in compact, solid-state depth sensing may be integrated in a future, autonomous inspection system. Aside from a simple cropping operation, the depth data is assessed "as-is", e.g., with the assumption that the raw depth data should be readily available for tasks such as odometry, motion planning, and hazard avoidance.

The proposed work has investigated the performance of depth sensors under different lighting conditions and in the presence of dirty water in the pipe. There are, however, numerous other conditions that may occur during an inspection of sewer pipes [81] such as intruding roots, chiseled connections, blockages, leakages, and structural defects. While this study compares the sensors in non-damaged pipes, the presented results may provide directions on the performance of the depth sensors in more challenging scenarios.

Author Contributions: Conceptualization, C.H.B.; methodology, C.H.B., A.S.J. and J.W.H.; software, C.H.B. and A.S.J.; validation, C.H.B. and T.B.M.; formal analysis, C.H.B.; investigation, C.H.B., A.S.J., J.W.H. and M.P.P.; resources, C.H.B. and A.S.J.; data curation, C.H.B.; writing-original draft preparation, C.H.B. and M.P.P.; writing—review and editing, C.H.B., A.S.J., M.P.P., K.N. and T.B.M.; visualization, C.H.B. and M.P.P.; supervision, C.H.B. and T.B.M.; project administration, C.H.B. and T.B.M.; funding acquisition, T.B.M. All authors have read and agreed to the published version of the manuscript.

Funding: This research was funded by Innovation Fund Denmark [grant number 8055-00015A] and is part of the Automated Sewer Inspection Robot (ASIR) project.

Institutional Review Board Statement: Not applicable.

Informed Consent Statement: Not applicable.

Data Availability Statement: Not applicable.

Conflicts of Interest: The authors declare no conflict of interest. The funders had no role in the design of the study; in the collection, analyses, or interpretation of data; in the writing of the manuscript; or in the decision to publish the results. 


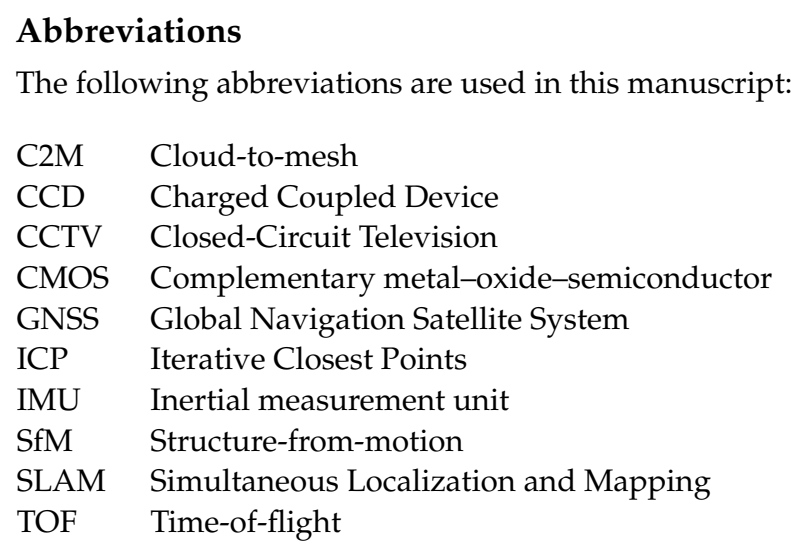

\section{References}

1. American Society of Civil Engineers (ASCE). 2021 Infrastructure Report Card-Wastewater. 2021. Available online: https: / /infrastructurereportcard.org/wp-content/uploads/2020/12/Wastewater-2021.pdf (accessed on 5 April 2021).

2. Statistisches Bundesamt. Öffentliche Wasserversorgung und öffentliche Abwasserentsorgung—Strukturdaten zur Wasserwirtschaft 2016; Technical Report; Fachserie 19 Reihe 2.1.3.; Statistisches Bundesamt: Wiesbaden, Gemany, 2018.

3. Berger, C.; Falk, C.; Hetzel, F.; Pinnekamp, J.; Ruppelt, J.; Schleiffer, P.; Schmitt, J. Zustand der Kanalisation in DeutschlandErgebnisse der DWA-Umfrage 2020. Korrespondenz Abwasser Abfall 2020, 67, 939-953.

4. IBAK. Available online: http://www.ibak.de (accessed on 5 April 2021).

5. $\quad$ Reverte, C.F.; Thayer, S.M.; Whittaker, W.; Close, E.C.; Slifko, A.; Hudson, E.; Vallapuzha, S. Autonomous Inspector Mobile Platform. U.S. Patent 8,024,066, 20 September 2011.

6. Haurum, J.B.; Moeslund, T.B. A Survey on image-based automation of CCTV and SSET sewer inspections. Automat. Construct. 2020, 111, 103061. [CrossRef]

7. Kirchner, F.; Hertzberg, J. A prototype study of an autonomous robot platform for sewerage system maintenance. Autonom. Robot. 1997, 4, 319-331. [CrossRef]

8. Kolesnik, M.; Streich, H. Visual orientation and motion control of MAKRO-adaptation to the sewer environment. In Proceedings of the Seventh International Conference on the Simulation of Adaptive Behavior (SAB'02), Edinburgh, UK, 4-9 August 2002; pp. 62-29.

9. Nassiraei, A.A.; Kawamura, Y.; Ahrary, A.; Mikuriya, Y.; Ishii, K. Concept and design of a fully autonomous sewer pipe inspection mobile robot "kantaro". In Proceedings of the 2007 IEEE International Conference on Robotics and Automation, Rome, Italy, 10-14 April 2007; pp. 136-143.

10. Chataigner, F.; Cavestany, P.; Soler, M.; Rizzo, C.; Gonzalez, J.P.; Bosch, C.; Gibert, J.; Torrente, A.; Gomez, R.; Serrano, D. ARSI: An Aerial Robot for Sewer Inspection. In Advances in Robotics Research: From Lab to Market; Springer: Berlin/Heidelberg, Germany, 2020; pp. 249-274.

11. Alejo, D.; Mier, G.; Marques, C.; Caballero, F.; Merino, L.; Alvito, P. A ground robot solution for semi-autonomous inspection of visitable sewers. In ECHORD++: Innovation from LAB to MARKET; Springer: Berlin/Heidelberg, Germany, 2019.

12. Tur, J.M.M.; Garthwaite, W. Robotic devices for water main in-pipe inspection: A survey. J. Field Robot. 2010, 4, 491-508.

13. Rome, E.; Hertzberg, J.; Kirchner, F.; Licht, U.; Christaller, T. Towards autonomous sewer robots: The MAKRO project. Urban Water 1999, 1, 57-70. [CrossRef]

14. Totani, H.; Goto, H.; Ikeda, M.; Yada, T. Miniature 3D optical scanning sensor for pipe-inspection robot. In Microrobotics and Micromechanical Systems; International Society for Optics and Photonics: Bellingham, WA, USA, 1995; Volume 2593, pp. 21-29.

15. Kuntze, H.B.; Haffner, H. Experiences with the development of a robot for smart multisensoric pipe inspection. In Proceedings of the 1998 IEEE International Conference on Robotics and Automation (Cat. No. 98CH36146), Leuven, Belgium, 20 May 1998; Volume 2, pp. 1773-1778.

16. Kampfer, W.; Bartzke, R.; Ziehl, W. Flexible mobile robot system for smart optical pipe inspection. In Nondestructive Evaluation of Utilities and Pipelines II; International Society for Optics and Photonics: Bellingham, WA, USA, 1998; Volume 3398, pp. 75-83.

17. Lawson, S.W.; Pretlove, J.R. Augmented reality and stereo vision for remote scene characterization. In Telemanipulator and Telepresence Technologies VI; International Society for Optics and Photonics: Bellingham, WA, USA, 1999; Volume 3840, pp. 133-143.

18. Kolesnik, M.; Baratoff, G. 3D interpretation of sewer circular structures. In Proceedings of the 2000 ICRA, Millennium Conference, IEEE International Conference on Robotics and Automation, Symposia Proceedings (Cat. No. 00CH37065), San Francisco, CA, USA, 24-28 April 2000; Volume 2, pp. 1453-1458.

19. Kolesnik, M.; Baratoff, G. Online distance recovery for a sewer inspection robot. In Proceedings of the 15th International Conference on Pattern Recognition, ICPR-2000, Barcelona, Spain, 3-7 September 2000; Volume 1, pp. 504-507.

20. Lawson, S.W.; Pretlove, J.R.; Wheeler, A.C.; Parker, G.A. Augmented reality as a tool to aid the telerobotic exploration and characterization of remote environments. Pres. Teleoperat. Virtual Environ. 2002, 11, 352-367. [CrossRef] 
21. Nüchter, A.; Surmann, H.; Lingemann, K.; Hertzberg, J. Consistent 3D model construction with autonomous mobile robots. In Proceedings of the Annual Conference on Artificial Intelligence, Hamburg, Germany, 15-18 September 2003; Springer: Berlin/Heidelberg, Germany, 2003; pp. 550-564.

22. Yamada, H.; Togasaki, T.; Kimura, M.; Sudo, H. High-density 3D packaging sidewall interconnection technology for CCD micro-camera visual inspection system. Electron. Commun. Jpn. 2003, 86, 67-75. [CrossRef]

23. Orghidan, R.; Salvi, J.; Mouaddib, E.M. Accuracy estimation of a new omnidirectional 3D vision sensor. In Proceedings of the IEEE International Conference on Image Processing 2005, Genova, Italy, 14 September 2005.

24. Ma, Z.; Hu, Y.; Huang, J.; Zhang, X.; Wang, Y.; Chen, M.; Zhu, Q. A novel design of in pipe robot for inner surface inspection of large size pipes. Mech. Based Des. Struct. Mach. 2007, 35, 447-465. [CrossRef]

25. Kannala, J.; Brandt, S.S.; Heikkilä, J. Measuring and modelling sewer pipes from video. Mach. Vis. Appl. 2008, 19, 73-83. [CrossRef]

26. Wakayama, T.; Yoshizawa, T. Simultaneous measurement of internal and external profiles using a ring beam device. In Two-and Three-Dimensional Methods for Inspection and Metrology VI; International Society for Optics and Photonics: Bellingham, WA, USA, 2008; Volume 7066, p. 70660D.

27. Chaiyasarn, K.; Kim, T.K.; Viola, F.; Cipolla, R.; Soga, K. Image mosaicing via quadric surface estimation with priors for tunnel inspection. In Proceedings of the 2009 16th IEEE International Conference on Image Processing (ICIP), Cairo, Egypt, 7-10 November 2009; pp. 537-540.

28. Esquivel, S.; Koch, R.; Rehse, H. Reconstruction of sewer shaft profiles from fisheye-lens camera images. In Proceedings of the Joint Pattern Recognition Symposium, Jena, Germany, 9-11 September 2009; Springer: Berlin/Heidelberg, Germany, 2009; pp. 332-341.

29. Esquivel, S.; Koch, R.; Rehse, H. Time budget evaluation for image-based reconstruction of sewer shafts. In Real-Time Image and Video Processing 2010; International Society for Optics and Photonics: Bellingham, WA, USA, 2010; Volume 7724, p. 77240M.

30. Saenz, J.; Elkmann, N.; Walter, C.; Schulenburg, E.; Althoff, H. Treading new water with a fully automatic sewer inspection system. In Proceedings of the ISR 2010 (41st International Symposium on Robotics) and ROBOTIK 2010 (6th German Conference on Robotics), Munich, Germany, 7-9 June 2010; pp. 1-6.

31. Hansen, P.; Alismail, H.; Browning, B.; Rander, P. Stereo visual odometry for pipe mapping. In Proceedings of the 2011 IEEE/RSJ International Conference on Intelligent Robots and Systems, IEEE, San Francisco, CA, USA, 25-30 September 2011; pp. 4020-4025.

32. Zhang, Y.; Hartley, R.; Mashford, J.; Wang, L.; Burn, S. Pipeline reconstruction from fisheye images. J. WSCG 2011, 19, 49-57.

33. Buschinelli, P.D.; Melo, J.R.C.; Albertazzi, A., Jr.; Santos, J.M.; Camerini, C.S. Optical profilometer using laser based conical triangulation for inspection of inner geometry of corroded pipes in cylindrical coordinates. In Optical Measurement Systems for Industrial Inspection VIII; International Society for Optics and Photonics: Bellingham, WA, USA, 2013; Volume 8788, p. 87881H.

34. Ying, W.; Cuiyun, J.; Yanhui, Z. Pipe defect detection and reconstruction based on 3D points acquired by the circular structured light vision. Adv. Mech. Eng. 2013, 5, 670487. [CrossRef]

35. Kawasue, K.; Komatsu, T. Shape measurement of a sewer pipe using a mobile robot with computer vision. Int. J. Adv. Robot. Syst. 2013, 10, 52. [CrossRef]

36. Larson, J.; Okorn, B.; Pastore, T.; Hooper, D.; Edwards, J. Counter tunnel exploration, mapping, and localization with an unmanned ground vehicle. In Unmanned Systems Technology XVI; International Society for Optics and Photonics: Bellingham, WA, USA, 2014; Volume 9084, p. 90840Q.

37. Arciniegas, J.R.; González, A.L.; Quintero, L.; Contreras, C.R.; Meneses, J.E. Three-dimensional shape measurement system applied to superficial inspection of non-metallic pipes for the hydrocarbons transport. In Dimensional Optical Metrology and Inspection for Practical Applications III; International Society for Optics and Photonics: Bellingham, WA, USA, 2014; Volume 9110, p. $91100 \mathrm{U}$.

38. Dobie, G.; Summan, R.; MacLeod, C.; Pierce, G.; Galbraith, W. An automated miniature robotic vehicle inspection system. In AIP Conference Proceedings; American Institute of Physics: College Park, MD, USA, 2014; Volume 1581, pp. 1881-1888.

39. Aghdam, H.H.; Kadir, H.A.; Arshad, M.R.; Zaman, M. Localizing pipe inspection robot using visual odometry. In Proceedings of the 2014 IEEE International Conference on Control System, Computing and Engineering (ICCSCE 2014), IEEE, Penang, Malaysia, 28-30 November 2014; pp. 245-250.

40. Skinner, B.; Vidal-Calleja, T.; Miro, J.V.; De Bruijn, F.; Falque, R. 3D point cloud upsampling for accurate reconstruction of dense $2.5 \mathrm{D}$ thickness maps. In Proceedings of the Australasian Conference on Robotics and Automation, ACRA, Melbourne, VIC, Australia, 2-4 December 2014.

41. Striegl, P.; Mönch, K.; Reinhardt, W. Untersuchungen zur Verbesserung der Aufnahmegenauigkeit von Abwasserleitungen. ZfVZeitschrift Geodäsie Geoinformation Landmanagement 2014. Available online: https://www.unibw.de/geoinformatik/publikationenund-vortraege/pdf-dateien-wissenschaftliche-publikationen/zfv_2014_2_striegl_moench_reinhardt.pdf (accessed on 5 April 2021).

42. Bellés, C.; Pla, F. A Kinect-Based System for 3D Reconstruction of Sewer Manholes. Comput. Aided Civ. Infrastruct. Eng. 2015, 30, 906-917. [CrossRef]

43. Hansen, P.; Alismail, H.; Rander, P.; Browning, B. Visual mapping for natural gas pipe inspection. Int. J. Robot. Res. 2015, 34, 532-558. [CrossRef] 
44. Wu, T.; Lu, S.; Tang, Y. An in-pipe internal defects inspection system based on the active stereo omnidirectional vision sensor. In Proceedings of the 2015 12th International Conference on Fuzzy Systems and Knowledge Discovery (FSKD), Zhangjiajie, China, 15-17 August 2015; pp. 2637-2641.

45. Huynh, P.; Ross, R.; Martchenko, A.; Devlin, J. Anomaly inspection in sewer pipes using stereo vision. In Proceedings of the 2015 IEEE International Conference on Signal and Image Processing Applications (ICSIPA), Kuala Lumpur, Malaysia, 19-21 October 2015; pp. 60-64.

46. Huynh, P.; Ross, R.; Martchenko, A.; Devlin, J. 3D anomaly inspection system for sewer pipes using stereo vision and novel image processing. In Proceedings of the 2016 IEEE 11th Conference on Industrial Electronics and Applications (ICIEA), Hefei, China, 5-7 June 2016; pp. 988-993.

47. Elfaid, A. Optical Sensors Development for NDE Applications Using Structured Light and 3D Visualization. Ph.D. Thesis, University of Colorado Denver, Denver, CO, USA, 2016.

48. Stanić, N.; Lepot, M.; Catieau, M.; Langeveld, J.; Clemens, F.H. A technology for sewer pipe inspection (part 1): Design, calibration, corrections and potential application of a laser profiler. Automat. Const. 2017, 75, 91-107. [CrossRef]

49. Nieuwenhuisen, M.; Quenzel, J.; Beul, M.; Droeschel, D.; Houben, S.; Behnke, S. ChimneySpector: Autonomous MAV-based indoor chimney inspection employing 3D laser localization and textured surface reconstruction. In Proceedings of the 2017 International Conference on Unmanned Aircraft Systems (ICUAS), Miami, FL, USA, 13-16 June 2017; pp. 278-285.

50. Yang, Z.; Lu, S.; Wu, T.; Yuan, G.; Tang, Y. Detection of morphology defects in pipeline based on 3D active stereo omnidirectional vision sensor. IET Image Process. 2017, 12, 588-595. [CrossRef]

51. Mascarich, F.; Khattak, S.; Papachristos, C.; Alexis, K. A multi-modal mapping unit for autonomous exploration and mapping of underground tunnels. In Proceedings of the 2018 IEEE Aerospace Conference, IEEE, Big Sky, MT, USA, 3-10 March 2018; pp. 1-7.

52. Reyes-Acosta, A.V.; Lopez-Juarez, I.; Osorio-Comparán, R.; Lefranc, G. 3D pipe reconstruction employing video information from mobile robots. Appl. Soft Comput. 2019, 75, 562-574. [CrossRef]

53. Zhang, X.; Zhao, P.; Hu, Q.; Wang, H.; Ai, M.; Li, J. A 3D Reconstruction Pipeline of Urban Drainage Pipes Based on MultiviewImage Matching Using Low-Cost Panoramic Video Cameras. Water 2019, 11, 2101. [CrossRef]

54. Jackson, W.; Dobie, G.; MacLeod, C.; West, G.; Mineo, C.; McDonald, L. Error analysis and calibration for a novel pipe profiling tool. IEEE Sens. J. 2019, 20, 3545-3555. [CrossRef]

55. Chen, X.; Zhou, F.; Chen, X.; Yang, J. Mobile visual detecting system with a catadioptric vision sensor in pipeline. Optik 2019, 193, 162854. [CrossRef]

56. Oyama, A.; Iida, H.; Ji, Y.; Umeda, K.; Mano, Y.; Yasui, T.; Nakamura, T. Three-dimensional Mapping of Pipeline from Inside Images Using Earthworm Robot Equipped with Camera. IFAC-PapersOnLine 2019, 52, 87-90. [CrossRef]

57. Nahangi, M.; Czerniawski, T.; Haas, C.T.; Walbridge, S. Pipe radius estimation using Kinect range cameras. Automat. Construct. 2019, 99, 197-205. [CrossRef]

58. Piciarelli, C.; Avola, D.; Pannone, D.; Foresti, G.L. A vision-based system for internal pipeline inspection. IEEE Trans. Indust. Inform. 2018, 15, 3289-3299. [CrossRef]

59. Fernandes, R.; Rocha, T.L.; Azpúrua, H.; Pessin, G.; Neto, A.A.; Freitas, G. Investigation of Visual Reconstruction Techniques Using Mobile Robots in Confined Environments. In Proceedings of the 2020 Latin American Robotics Symposium (LARS), 2020 Brazilian Symposium on Robotics (SBR) and 2020 Workshop on Robotics in Education (WRE), Natal, Brazil, 9-13 November 2020; pp. 1-6.

60. Chuang, T.Y.; Sung, C.C. Learning and SLAM Based Decision Support Platform for Sewer Inspection. Remote Sens. 2020, $12,968$. [CrossRef]

61. Kolvenbach, H.; Wisth, D.; Buchanan, R.; Valsecchi, G.; Grandia, R.; Fallon, M.; Hutter, M. Towards autonomous inspection of concrete deterioration in sewers with legged robots. J. Field Robot. 2020, 37, 1314-1327. [CrossRef]

62. Yoshimoto, K.; Watabe, K.; Tani, M.; Fujinaga, T.; Iijima, H.; Tsujii, M.; Takahashi, H.; Takehara, T.; Yamada, K. Three-Dimensional Panorama Image of Tubular Structure Using Stereo Endoscopy. Available online: www.ijicic.org/ijicic-160302.pdf (accessed on 5 April 2021).

63. Zhang, G.; Shang, B.; Chen, Y.; Moyes, H. SmartCaveDrone: 3D cave mapping using UAVs as robotic co-archaeologists. In Proceedings of the 2017 International Conference on Unmanned Aircraft Systems (ICUAS), Miami, FL, USA, 13-16 June 2017; pp. 1052-1057.

64. Godard, C.; Mac Aodha, O.; Brostow, G.J. Unsupervised monocular depth estimation with left-right consistency. In Proceedings of the IEEE Conference on Computer Vision and Pattern Recognition, Honolulu, HI, USA, 21-26 July 2017; pp. 270-279.

65. Godard, C.; Mac Aodha, O.; Firman, M.; Brostow, G.J. Digging into self-supervised monocular depth estimation. In Proceedings of the IEEE/CVF International Conference on Computer Vision, Seoul, Korea, 27 October-2 November 2019; pp. 3828-3838.

66. Tölgyessy, M.; Dekan, M.; Chovanec, L.; Hubinskỳ, P. Evaluation of the Azure Kinect and Its Comparison to Kinect V1 and Kinect V2. Sensors 2021, 21, 413. [CrossRef]

67. Chen, S.; Chang, C.W.; Wen, C.Y. Perception in the dark—Development of a ToF visual inertial odometry system. Sensors 2020, 20, 1263. [CrossRef]

68. Khattak, S.; Papachristos, C.; Alexis, K. Vision-depth landmarks and inertial fusion for navigation in degraded visual environments. In Proceedings of the International Symposium on Visual Computing, Las Vegas, NV, USA, 19-21 November 2018; Springer: Berlin/Heidelberg, Germany, 2018; pp. 529-540. 
69. pmdtechnologies ag. Development Kit BriefCamBoard Pico Flexx. Available online: https://pmdtec.com/picofamily/wp-content/ uploads/2018/03/PMD_DevKit_Brief_CB_pico_flexx_CE_V0218-1.pdf (accessed on 8 January 2021).

70. Intel. Intel囚RealSense ${ }^{\mathrm{TM}} D 400$ Series Product Family_Datasheet. Available online: https://www.intel.com/content/dam/support/ us/en/documents/emerging-technologies/intel-realsense-technology/Intel-RealSense-D400-Series-Datasheet.pdf (accessed on 8 January 2021).

71. Smart Vision Lights. S75 Brick Light Spot Light. Available online: https://smartvisionlights.com/wp-content/uploads/S75_ Datasheet-2.pdf (accessed on 5 April 2021).

72. pmdtechnologies ag, Am Eichengang 50, 57076 Siegen, Germany. CamBoard pico flexx—Getting Started; 2018.

73. Javaheri, A.; Brites, C.; Pereira, F.; Ascenso, J. Subjective and objective quality evaluation of 3D point cloud denoising algorithms. In Proceedings of the 2017 IEEE International Conference on Multimedia \& Expo Workshops (ICMEW), Hong Kong, China, 10-14 July 2017; pp. 1-6.

74. Cignoni, P.; Rocchini, C.; Scopigno, R. Metro: Measuring Error on Simplified Surfaces; Computer graphics forum; Wiley: Hoboken, NJ, USA, 1998; Volume 17, pp. 167-174.

75. Su, H.; Duanmu, Z.; Liu, W.; Liu, Q.; Wang, Z. Perceptual quality assessment of 3D point clouds. In Proceedings of the 2019 IEEE International Conference on Image Processing (ICIP), Taipei, Taiwan, 22-25 September 2019; pp. 3182-3186.

76. Mekuria, R.; Blom, K.; Cesar, P. Design, implementation, and evaluation of a point cloud codec for tele-immersive video. IEEE Trans. Circ. Syst. Video Technol. 2016, 27, 828-842. [CrossRef]

77. Lague, D.; Brodu, N.; Leroux, J. Accurate 3D comparison of complex topography with terrestrial laser scanner: Application to the Rangitikei canyon (NZ). ISPRS J. Photogram. Remote Sens. 2013, 82, 10-26. [CrossRef]

78. Girardeau-Montaut, D. CloudCompare. 2016. Available online: https:/ /www.danielgm.net/cc (accessed on 5 April 2021).

79. Schnabel, R.; Wahl, R.; Klein, R. Efficient RANSAC for Point-Cloud Shape Detection; Computer graphics forum; Wiley: Hoboken, NJ, USA, 2007, Volume 26, pp. 214-226.

80. Elma Instruments A/S. Manual Elma 1335.

81. Haurum, J.B.; Moeslund, T.B. Sewer-ML: A Multi-Label Sewer Defect Classification Dataset and Benchmark. arXiv 2021, arXiv:2103.10895 